\title{
Prediction of single salt rejection in nanofiltration membranes by independent measurements.
}

\author{
Verónica SILVA ${ }^{1}$, Miguel MONTALVILLO ${ }^{1}$, Francisco Javier CARMONA ${ }^{2}$, Laura \\ PALACIO ${ }^{l}$, Antonio HERNÁNDEZ ${ }^{l}$ and Pedro PRÁDANOS ${ }^{1 *}$ \\ ${ }^{1}$ Grupo de Superficies y Materiales Porosos, Dpto. Física Aplicada, Facultad de Ciencias, \\ Universidad de Valladolid, 47071Valladolid, Spain \\ ${ }^{2}$ Dpto. de Física Aplicada. Escuela Politécnica, Universidad de Extremadura, 10004 Cáceres, \\ Spain \\ *E-mail: pradanos@termo.uva.es
}

\begin{abstract}
In this work a method is proposed to predict salt rejection by nanofiltration. The procedure starts from the steric, electric and dielectric exclusion model with charge (and permitivity) depending on the concentration along the pore, SEDE-VCh, for membrane characterization, and substitutes all fitting parameters by values obtained by independent methods. These parameters are the relative permittivity inside the pores and the two constants of the Freundlich isotherm for the volumetric charge density, which can be obtained by impedance spectroscopy techniques. Moreover, the pore size and shape and the active layer thickness are required to complement the model. The pore size was obtained by using a neutral solute rejection test and the active layer thickness was estimated by SEM. Therefore, the model also requires pore shape as input. AFM measurements suggest the assumption of a slit shape for the pores.

A Desal-HL membrane has been structurally, electrically and functionally characterized. These data allowed the testing of the predictive model that was subsequently demonstrated; as far as results are good enough considering the complexity of the mechanisms involved. Consequently, it seems clear that once the model parameters have been obtained by independent methods, it can be used as a predictive tool.
\end{abstract}

Keywords: Impedance Spectroscopy, Nanofiltration, Membrane Potential, Transport numbers, Dielectric properties 
1. Introduction

Nanofiltration (NF) membranes possess some special characteristics that distinguish them from ultrafiltration (UF) and reverse osmosis (RO) ones. Firstly, they keep relatively high permeate flux at low pressure operation compared with conventional RO [1], and secondly, most of them are electrically charged with the subsequent effect on the solute separation mechanism.

Due to the clear interest of NF, it is desirable to have a way to estimate the performance of NF membranes for different solutes and/or combinations of solutes in order to have a predictive understanding of their behavior. As a consequence, there have been many efforts, with this aim in mind, focusing on the development and optimization of mathematical models to predict the separation properties of NF membranes. Firstly based on irreversible thermodynamics (Kedem, Katchalsky and Spiegler works) [2, 3], continuing with the hydrodynamic model or pore model introduced by Ferry [4], and the development of hydrodynamic approach models based on the extended Nernst-Planck equation such as the steric hindrance pore, SHP, [5], Teorell-Meyer Sievers, TMS, [6, 7], the space charge model, SCPM, by Wang et al.[8] and more recently the Donnan steric partitioning model, DSPM, which combines the steric and Donnan exclusion effects [9].

Nowadays the most complete models include dielectric exclusion effect [10], including steric, Donnan and dielectric partitioning effects in the interfaces and convective, diffusive and electromigrative transport effects in the inner part of the membrane. The mass transfer through the membrane is described using the extended Nernst-Planck equation modified by hydrodynamic coefficients to reflect the influence of the pore constriction on both convection and diffusion. The equilibrium partitioning relation takes into account electric and dielectric effects to describe the distribution of species at the pore inlet and outlet 
[1]. These effects are the Donnan exclusion and the dielectric exclusion, being the later composed by two terms, the Born effect and the image forces one. The Born effect is connected with the low values of the relative permittivity of a liquid inside a pore of nanometer dimensions. The image forces effect correspond to the interaction between the ions and the polarization charges induced by them at the pore wall.

Bandini in 2001-2002 firstly presented the Donnan steric partitioning model with Dielectric exclusion model, DSPM\&DE, [11]. It is a model in which the ionic partitioning at the interfaces between the membrane and the external phase takes into account the three separation mechanisms: steric, Donnan equilibrium and dielectric exclusion. Bandini's model introduced the idea of the dielectric exclusion as an additional cause of partitioning to those of bare Donnan steric pore model (DSPM) initially proposed by Bowen $[9,12,13]$. We refer to the reading of the work of Bandini for a more extensive explanation of the model [14].

In 2005, Szymczyk and Fievet proposed another model, the steric, electric and dielectric exclusion, SEDE, model [15]. The volume charge density of a NF membrane was determined from tangential streaming potential measurements (TSP) and the model was used to assess the rejection rate of the membrane with a single adjustable parameter: the relative permittivity of the solution filling the pores. In a later work [1] Lanteri et al. proved that the SEDE model is able to reproduce both experimental rejection rates and membrane potentials by using several fitting parameters: effective pore size, effective thickness-to-porosity ratio, $\Delta x_{\mathrm{a}} / A_{\mathrm{ka}}$, effective volume charge density, $X$, and relative permittivity inside the pores, $\varepsilon_{\mathrm{p}}$, all them being considered constant through the membrane. Unfortunately, it was observed that there are different couples of values $\left(X, \varepsilon_{\mathrm{p}}\right)$ that lead to the same membrane potential value, between all of them, true values of $X$ and $\varepsilon_{\mathrm{p}}$ are difficult to obtain by any fitting procedure with reasonable physical meaning for both the parameters. In a continuing work 
[16], Déon et al. assumed the model proposed by Silva et al. [17] that considered that the charge density within the pore varies with concentration. Déon et al. did not included the image force term into the dielectric effect. However they assumed that this effect would be indirectly included in the "effective" estimated value for $\varepsilon_{\mathrm{p}}$ that can be obtained by fitting but that sometimes lead to weird values.

This article presents a novel method to predict the salt rejection developed by a NF membrane. The model includes three parameters: the relative permittivity inside the pores, $\varepsilon_{p}$, and the $\gamma$ and $\Gamma$ parameters of a Freundlich charge isotherm of the volumetric charge density, $X=\Upsilon_{c} c^{\Gamma}$. Unlike the papers presented so far, in the present work these three parameters are obtained by independent methods or experimental techniques.

In this case, an estimation of the thickness of the active membrane layer, $\Delta x_{\mathrm{a}}$, is obtained from scanning electron microscopy, SEM, allowing the evaluation of membrane porosity, $A_{\mathrm{k}}$, from water permeability measurements. The membrane porosity of the active layer is necessary to link the relative permittivity of the wet membrane with the corresponding value for the solution inside the pores and the dry membrane material, as it will be explained later. Transport numbers are obtained from membrane potential measurements. The viscosity inside the pore is calculated by using only the pore radius and the bulk value. In the present work, as it was done previously [17], the volumetric charge density and the relative permittivity inside the pores were considered as variable along the pores and depending on concentration, $\varepsilon_{\mathrm{p}}=f(c) \quad X=f(c)$. These two magnitudes are obtained from Impedance Spectroscopy (IS) measurements using a similar method to that described previously [10]. The model can be called SEDE-VCh model because it uses steric, electric and dielectric exclusion with $X$ (and $\varepsilon_{\mathrm{p}}$ ) assumed as depending on concentration (an 
consequently on distance along the pore). The changes of charge along the pore, within this model, can be as large as to span over an order of magnitude, depending on the operation conditions and leads better fitting to the experimental results [17].

The main objective of the present work is to evaluate the predictive capacity of the model to foretell NF performances. The predictive character of the model consists in its ability to obtain retention from independently known morphological and electrical properties of the membrane. This permits securing the proposed model as to get membrane retention by easier and faster procedures tan the simple measurement of observed retention followed by a careful concentration-polarization through mass transfer models. With this aim, the experimental volume flux and intrinsic retention of aqueous $\mathrm{NaCl}$ solutions through a flat sheet Desal HL, a polyamide NF membrane made by GE-Osmonics, will be compared with the corresponding predictions obtained from independently measured $\varepsilon_{\mathrm{p}}$ and $X$. It will be shown that fair accordance is found for the concentrations range studied.

Desal HL is a typical composite membrane, it consists of three layers: a thin top selective polyamide layer of a few hundred nanometers in thickness (poly(piperazineamide)), an asymmetric microporous polysulfone support layer, and a polyester non-woven fabric layer for mechanical strength $[18,19]$. This membrane has been studied in our previous work [10, 20-22] and others authors [18, 19, 23, 24] so there is a good reservoir of knowledge on its characteristics and functionality that can help to assess the models and its predictive capacity.

\section{THEORY}

\subsection{Dielectric Analysis}

Impedance spectroscopy measurements determine the electrical impedance of a system as a function of frequency. When the objective is the electrical characterization of a 
membrane, the system is formed by five elements or layers: electrode + electrolyte + membrane + electrolyte + electrode. The system corresponds to three phases: electrode, membrane and electrolyte. In such a system it is possible to recognize a scheme of series resistance as shown in Figure 1. Evidently similar layers can be characterized by a unique set of electrical parameters or elements.

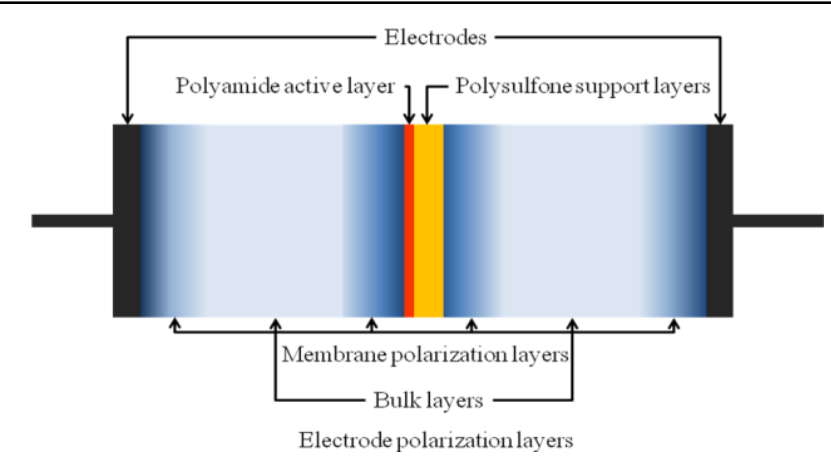

Figure 1: Schematic representation of the impedance measurement system.

For the dielectric analysis, we follow the same procedure than in our previous work, in order to analyse the Impedance Spectroscopy results [10, 21]. A summary of the procedure followed can be found in the Appendix A.

\subsection{Relative permittivity and conductivity inside NF pores}

The relative permittivity of the wet membrane, $\varepsilon_{\text {memb }}$ (as obtained from Equation (A.8) in Table A.1) can be split as a linear combination of two terms, the permittivity inside the pores, $\varepsilon_{\mathrm{p}}$, and the membrane dry material permittivity, $\varepsilon_{\mathrm{d}}$, as,

$$
\varepsilon_{\mathrm{memb}}=\varepsilon_{\mathrm{p}} A_{\mathrm{ka}}+\varepsilon_{\mathrm{d}}\left(1-A_{\mathrm{ka}}\right)
$$

The same relation is applicable for the overall or wet conductivity, $\kappa_{\text {memb }}$ :

$$
\kappa_{\mathrm{memb}}=\kappa_{\mathrm{p}} A_{\mathrm{ka}}+\kappa_{\mathrm{d}}\left(1-A_{\mathrm{ka}}\right)
$$


Where $A_{\mathrm{ka}}$ is the porosity of the membrane active layer. $A_{\mathrm{ka}}$ can be estimated from $\Delta x_{\mathrm{a}} / A_{\mathrm{ka}}$ if the mean membrane thickness is known. This ratio can be obtained from the Hagen-Poiseuille equation assuming slit pores and viscosity correction [25] as,

$$
\frac{\Delta x_{\mathrm{a}}}{A_{\mathrm{ka}}}=\frac{r_{\mathrm{p}}^{2}}{3 \eta_{\mathrm{p}, \mathrm{slit}} L_{\mathrm{w}}}
$$

Where $\eta_{\mathrm{p}, \text { slit }}$ is the viscosity inside pores and $L_{\mathrm{w}}$ is the water permeability. $r_{\mathrm{p}}$ is the pore radius (for slit pores $r_{\mathrm{p}}=h / 2$, with $h$ the thickness of the slit). It is known that viscosity inside very narrow pores is enhanced due to the effects of confinement, here this effect has been calculated for slit-shaped pores as proposed by Wesolowska [25]:

$$
\eta_{\mathrm{p}, \mathrm{sit}}=\frac{10 \eta_{\mathrm{b}}}{1+9\left(1-d / r_{\mathrm{p}}\right)^{3}}
$$

$\eta_{\mathrm{b}}$ is the bulk water viscosity and $d$ is the thickness of the layer of water fully oriented towards the walls of the pores with a viscosity ten times the value for the bulk [10]. The existance of such a high viscosity on the pore walls may seem improbable but seems to be experimentally confirmed as discussed by Bowen and Welfoot [26, 27].

\subsection{Thermodynamic equilibrium at the interfaces}

Due to the nature of the impedance spectroscopy measurements, there is neither any applied pressure nor any concentration gradient between both sides of the membrane. The corresponding profiles in both impedance spectroscopy and permeation experiments are shown in Figure 2. 
Figure 2: Scheme of concentration profile in the pores of the active layer of the membrane, a) on the experiences of Impedance Spectroscopy, b) on the experiences of NF of a solution.

Consequently the only mechanisms causing separation of the electrolyte are those related to the thermodynamic equilibrium in both interfaces which are can be calculated as $[26,27]:$

$$
\frac{c_{i, 0}}{c_{i, \mathrm{~m}}}=\phi_{i} \frac{\gamma_{i, \mathrm{~m}}}{\gamma_{i, 0}} \exp \left(-z_{i} \Delta \Psi\right) \exp \left(-\Delta W_{i, \mathrm{Borm}}^{\prime}\right) \exp \left(-\Delta W_{i, \mathrm{~m}}^{\prime}\right)
$$

were $c_{i, 0}$ and $c_{i, \mathrm{~m}}$ are the concentration inside and outside the membrane, respectively, $\gamma_{i, 0}$ and $\gamma_{i, \mathrm{~m}}$ are the corresponding activity coefficients, $\phi_{i}$, the steric factor, takes into account the steric effect, $\Delta \Psi$ represents the normalized Donnan potential, and the dielectric effects are considered thorough the Born term, $\Delta W_{i, \text { Born }}^{\prime}$ [28], and the image forces effects $\Delta W_{i, \text { im }}^{\prime}[27,29,30]$. The expressions for these dielectric effects are shown in appendix $\mathrm{B}$ (Table B.1). Since we are working with dilute solutions, the activity coefficients are considered approximately 1 . Note that, for slit pores: $\phi_{i}=\left(1-\lambda_{i}\right)$, with $\lambda_{i}=r_{i, \text { Stokes }} / r_{\mathrm{p}}$, being $r_{i, \text { Stokes }}$ the Stokes radius of $i$-ion.

Changes in conductivity from inside to outside the membrane can be correlated with the equilibrium conditions as done in a previous work [10] through the following relation: 


$$
\frac{\kappa_{\mathrm{p}}}{\kappa_{\mathrm{b}}}=\frac{U_{1, \mathrm{p}} t_{1, \mathrm{~b}}}{U_{1, \mathrm{~b}} t_{1, \mathrm{p}}}\left(\sqrt{\left(\frac{X}{2 c_{\mathrm{b}}}\right)^{2}+\theta_{1} \theta_{2}}+\left(2 t_{1, \mathrm{p}}-1\right) \frac{X}{2 c_{\mathrm{b}}}\right)
$$

Where $\theta_{\mathrm{i}}$ is a coefficient grouping the influence of steric and dielectric effects and can be written as:

$$
\theta_{i}=\phi_{i} \exp \left(-\Delta W_{i, \text { Bor }}^{\prime}\right) \exp \left(-\Delta W_{i, \text { im }}^{\prime}\right)
$$

The subscripts 1 and 2 refer to cation and anion respectively and $\mathrm{p}$ and $\mathrm{b}$ indexes refer to the concentration just inside and outside the membrane interface respectively (p: pores, b: bulk). Parameters $U_{1, \mathrm{p}} t_{1, \mathrm{p}}$ and $U_{1, \mathrm{~b}} t_{1, \mathrm{~b}}$ are the cation mobilities and transport numbers inside and outside the membrane, respectively. Transport numbers are known to represent the fraction of the total current carried by the positive and negative ions.

It is well-known that the adsorption process of ions in NF membranes can be successfully described by a Freundlich isotherm $[31,32]$.

$$
X=r_{c}{ }^{\Gamma}
$$

Substituting this isotherm into Equation (6), we get:

$$
\frac{\kappa_{\mathrm{p}}}{\kappa_{\mathrm{b}}}=\frac{U_{1, \mathrm{p}} t_{1, \mathrm{~b}}}{U_{1, \mathrm{~b}} t_{1, \mathrm{p}}}\left(\sqrt{\left(\frac{\gamma c_{\mathrm{b}}^{\Gamma}}{2 c_{\mathrm{b}}}\right)^{2}+\theta_{1} \theta_{2}}+\left(2 t_{1, \mathrm{p}}-1\right) \frac{\left(\gamma c_{\mathrm{b}}^{\Gamma}\right)}{2 c_{\mathrm{b}}}\right)
$$

In this equation there are three parameters to fit which can be assumed as independent of concentration: $r, \Gamma$ and the ratio $U_{1, \mathrm{p}} / U_{1, \mathrm{~b}}$. Note that in order to use Equation (9), transport number should be known inside the pores. 


\subsection{SEDE-VCh model for NF experiments}

The SEDE-VCh model is the most complete approach based on the Nernst-Planck extended equation [12, 33-35] and is our aim here, as mentioned, to test its predictive features. In previous works, we demonstrated that the SEDE-VCh model can be used for the electrical characterization $\left(X, \varepsilon_{\mathrm{p}}\right)$ of NF membranes immersed in single salt solutions [17] and also for multi-component mixtures with a common ion [22].

One of the major uncertainties in the characterization of a NF membrane is the geometry of the cross section of the active layer pores. In most papers, authors assume two ideal situations: pores with cylindrical section or pores with slit shape $[22,36]$. In other cases, authors go for only one of the two geometries based on preceding knowledge from several characterization techniques $[37,38]$ or by fittings when mass transfer models are used $[15$, 39]. The model can be applied for cylindrical and slit pore geometries however, when cylindrical geometry was assumed, some unusual values of $\varepsilon_{\mathrm{p}}$ were found in the literature (bigger than $\left.\varepsilon_{\mathrm{w}}=78.5\right)[17,40]$ (both effects, Born and "images forces", were taken into account). When slit pore geometry is assumed, most of the results found in literature are in better agreement with what could be expected giving, in particular, dielectric constants inside

pores below the water bulk value $\left(\varepsilon_{\mathrm{p}}<\varepsilon_{\mathrm{w}}\right)$. However, $\varepsilon_{\mathrm{p}}$ values bigger than 78.5 have also been found when the charge density is evaluated from other techniques [41]. In the present work, the use of slit geometry is supported also by the AFM results in the membrane Surface, as will be explained in section 4.1.

To summarize the SEDE-VCh, we listed below the basis of the model together with the list of equations involved presented in appendix B (Table B.1).

i. Slit-shapes pores

ii. Volumetric charge density and permittivity depend on concentration 
iii. Variable volumetric charge density along the pores.

iv. Image charges forces effects and Born effects are considered.

v. No dielectric effects are considered due to dispersion interaction occurring between ions within pores and the membrane material [42], following most of the authors who use these models [43].

In Figure 3, a scheme of the procedure of evaluating the predictive power of the model is shown.

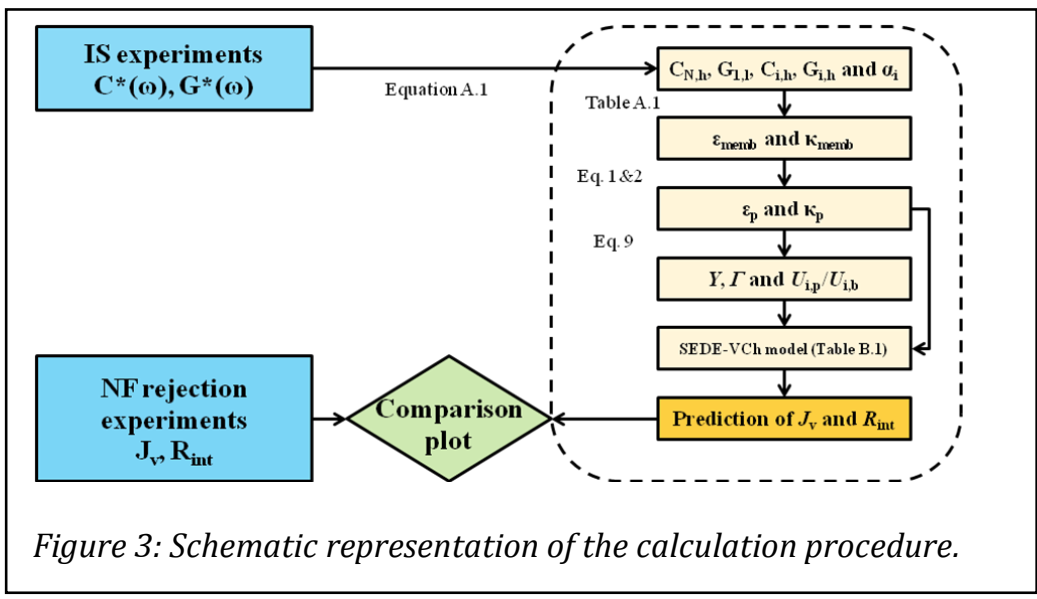

\section{EXPERIMENTAL}

\subsection{Membrane.}

A flat sheet commercial NF membrane Desal HL has been used. This is a polyamide membrane manufactured by GE-Osmonics (Minnetonka, MN, USA). According to the manufacturers, it has a MWCO between 150 and $300 \mathrm{~g} / \mathrm{mol}$, and it can be used in a pH range of 3-9 and up to a maximum temperature of $50^{\circ} \mathrm{C}$.

\subsection{Atomic Force Microscopy (AFM).}

Atomic Force Microscopy has been performed with a Nanoscope Multimode IIIa scanning probe microscope from Digital Instruments (Veeco Metrology Inc., Santa Barbara, CA, USA). As phase images show surface features with greater clarity than the height image 
alone, tapping mode was used [44, 45]. This technique allows the mapping of different components in polymeric materials.

For the tapping mode (intermittent contact), an electron beam deposited and sharpened tip was used; made by Nanotools (Nanotools, Munich, Germany) with a length of $1000 \mathrm{~nm}$, a point angle less than $10^{\circ}$ and sharpened with a radius of curvature always less than $5 \mathrm{~nm}$, according to the manufacturer specifications. Images have been obtained in ambient air with dry samples (as supplied by the manufacturer), and with samples previously wetted with water or ethanol.

\subsection{Water Permeability, Permeate Flux and Solute Retention}

Membrane permeability has been determined from experimental measures by the slope of the linear fit of the volumetric flux versus pressure data for the range from 1 to 5 MPa. The HP4750 stirred cell from Sterlitech (Sterlitech co, Kent, WA, USA) was used. Previously, the membrane was stabilized being immersed in water at $5 \mathrm{MPa}$ for one hour.

The neutral solutes retention measurements were performed with a $1 \mathrm{~g} / \mathrm{L}$ solution of tetraethylene glycol in water using the dead-end method. The same Sterlitech cell, used to determine the hydraulic permeability of the membrane, has been used for the retention experiments. The detailed procedure was previously described [20].

Retention measurements for charged solutes were performed using sodium chloride solutions. These experiments of retention and permeate flux were carried out in a flat sheet cross flow cell, Sepa CF from GE-Osmonics; fed with concentrations between 5 and 500 $\mathrm{mol} / \mathrm{m}^{3}$ and applied pressure difference from 10-50 bars. Retention results for the $\mathrm{NaCl}$ solutions have been published in a previous work [22], and those data are here used as published. 


\subsection{Impedance Spectroscopy}

Electrical characterization of the membrane was carried out by impedance spectroscopy technique. A circular membrane sample was placed between two flat and circular $\mathrm{Ag} / \mathrm{AgCl}$ electrodes of $32 \mathrm{~mm}$ of diameter. The holder cell has two identical methacrylate hemi-cells of $10.18 \mathrm{~cm}^{2}$ of active area. These two hemi-cells allow the continuous flow of identical solutions at both sides of the membrane, assuring the complete equilibrium between their faces. All components are located inside a stainless steel vessel that behaves like a Faraday shield and isolates the system from any external electromagnetic field. The cell and the whole arrangement were designed and built by us; and a more detailed description can be found in already published works [10, 21].

The non-woven support of the membrane has been removed from the membrane by mechanical peeling. Before the measurements, the membrane was conditioned during 24 hours inside the cell with Milli-Q (Millipore, Subsidiary of Merck KGaA, Billerica, MA, USA) deionized water, in order to remove air and impurities. With the membrane placed in the holder system, the solution has been kept flowing on each side of the membrane at the same flux $(0.6 \mathrm{~L} / \mathrm{min})$ and pressure, during a few minutes, to stabilize the system.

During the measurements, the solution was continuously flowing tangentially on both sides of the membrane, at the same rate of $0.6 \mathrm{~L} / \mathrm{min}$ and thermostated at $298 \pm 1 \mathrm{~K}$ by using a thermostatic bath.

Impedance measurements were taken using a Solartron 1260 (Ametek, Berwyn, PA, United States) in a frequency range from $10 \mathrm{MHz}$ to $10 \mathrm{mHz}$ and $10 \mathrm{mV}$ of applied $\mathrm{AC}$ voltage. The equipment is controlled by the commercial acquisition and control software from Solartron Analytical. Sampling was fixed at 7 points per decade, which gives 64 points per sample. This number of points is enough to appreciate all relaxation times and each measurement was finished in a reasonable period of one hour. These IS measurement was 
repeated using increasing concentrations of salt solutions, for a wide range between 0.01 and $10 \mathrm{~mol} / \mathrm{m}^{3}$ prepared from Milli-Q deionized water.

\subsection{Membrane potential.}

The Membrane Potential was determined by using the same membrane holder used for the impedance spectroscopy technique. Both sides were properly stirred by the recirculation of the solution with a water flux of $0.6 \mathrm{~L} / \mathrm{min}$ in order to reduce the concentration polarization effect. $\mathrm{A} \mathrm{Cl}^{-}$selective membrane electrode, ISE 9652 from Crison (Hach-Lange, Danaher Corporation, Washington, D.C.,_United States) has been placed at each side of the cell and connected to a high impedance voltmeter. The hydrostatic pressure has been kept equal in both sides of the cell by placing the solution reservoirs at the same height. The temperature has been kept at $298 \pm 1 \mathrm{~K}$ by using a thermostatic bath.

In a previous work it was experimentally demonstrated that the transport number for $\mathrm{KCl}$ solutions is practically constant in the concentration range under analysis [10]. Moreover, it was found that the transport number, for the same membrane used here, Desal HL, was not influenced by the membrane support. In order to test this assumption, membrane potential experiments were carried out with the active layer of the membrane facing the lowest concentration solution after immersion in the higher concentration solution and compared with measurements performed with the active layer facing the highest concentration solution after immersion in the lower concentration solution [10].

Assuming that for $\mathrm{NaCl}$, these two factors are also accomplished, a process for membrane potential measurements was designed, keeping constant the concentration in contact with the active layer of the membrane $\left(c_{\text {high }}=10 \mathrm{~mol} / \mathrm{m}^{3}\right)$ and varying the concentration values in contact with the support $\left(0.01 \mathrm{~mol} / \mathrm{m}^{3}<c_{\text {low }}<5 \mathrm{~mol} / \mathrm{m}^{3}\right)$. 
The electric potential difference measured by the electrodes through the membrane system is called cell potential, $E_{\text {cell }}$. This potential is related to the membrane potential as $E_{\text {memb }}=E_{\text {cell }}-E_{\text {Nernst }}[46]$. The $E_{\text {Nernst }}$ term is the potential difference between the solution of high concentration, $c_{\text {high }}$, and that of low concentration, $c_{\text {low }}$; and it corresponds to the Nernstian contribution due to the concentration differences in both the electrode-solution interfaces. This potential drop, $E_{\text {Nernst }}$, has been previously determined by measuring against a commercial $\mathrm{Ag} / \mathrm{AgCl}$ reference electrode (Ref. 5044 of Crison). Each electrode has been placed alternatively in the high and low concentration compartments united by a saline bridge to the other compartment containing the reference electrode. An average of both readings has been used in order to avoid effects of asymmetry.

\section{RESULTS AND DISCUSSION}

\subsection{Membrane Parameters.}

As mentioned, AFM can enlighten on the average pore section geometry and on the question on should be assumed circular or slit-shaped. As said in section 3, AFM in tapping mode was carried on membrane samples surrounded by air, first on absolutely dry surfaces, as supplied by the manufacturer, and afterwards on wet. They have been dipped in water and alcohol. Although ethanol is not involved in filtration experimental, it has been used in AFM characterization because its low surface tension facilitates the AFM measurements. Ethanol surface tension is $22.51 \mathrm{mN} \cdot \mathrm{m}^{-1}$ (at $25^{\circ} \mathrm{C}$ ), much lower that water's, $72.01 \mathrm{mN} \cdot \mathrm{m}^{-1}$ [47].

Figure 4 is an example of the so obtained AFM images. The two in the top row have been taken with the membrane as supplied by the manufacturer in air. The left image (Figure 4.a) displays the topography and the right picture (Figure 4.b) corresponds to the phase image. The two images in the bottom row were taken after the membrane was drenched in ethanol. Images obtained with water are very similar to those obtained with ethanol, but of 
Figure 5.a shows a scheme of the phase change process when the tip moves from hard to soft areas. Images $\mathrm{b}$ and $\mathrm{d}$ of Figure 4 show the phase change (in tapping mode) associated to the change in viscoelastic properties of the surface, providing additional information to the topographical projections [49].

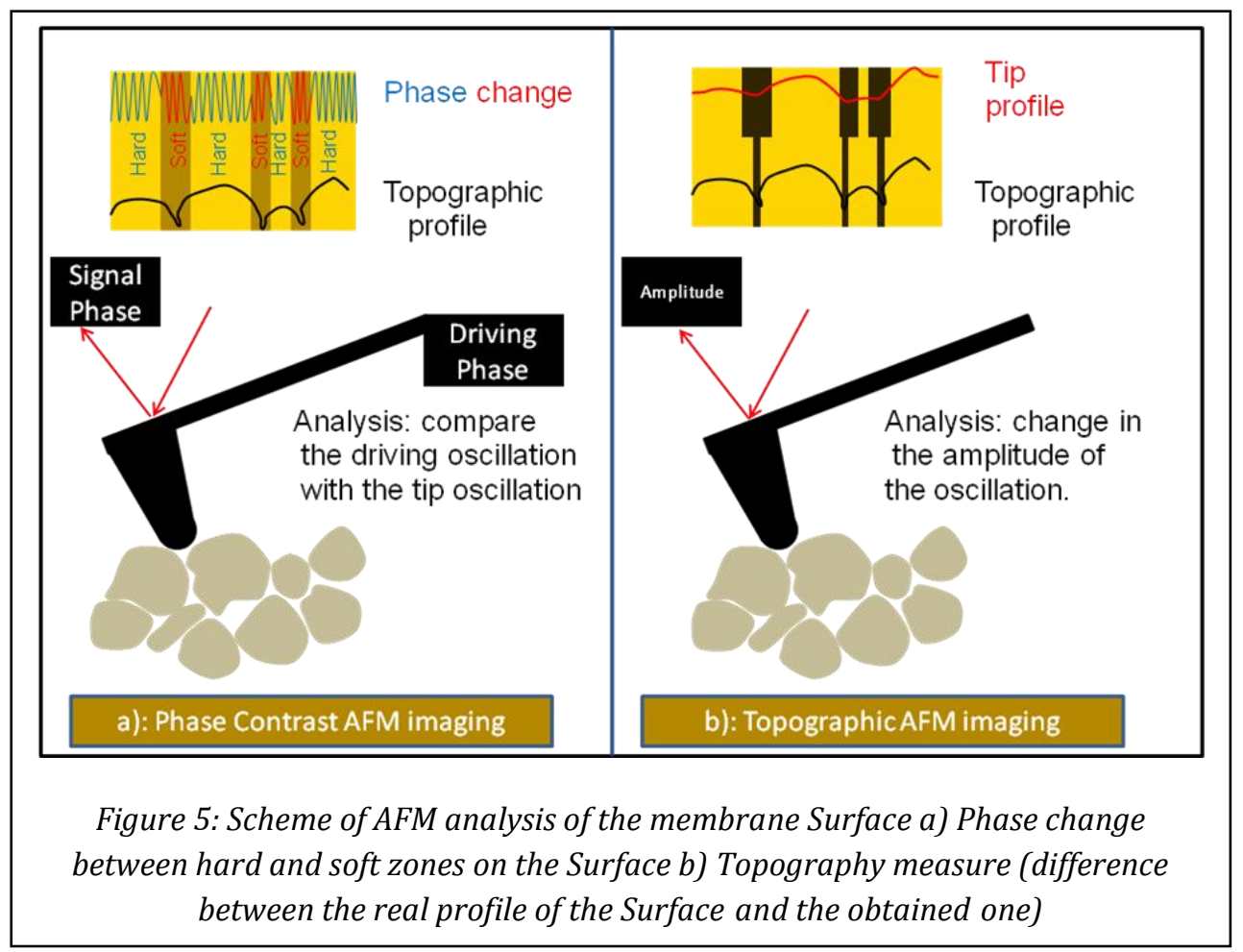

In the phase contrast images, especially for the wet sample, the polymer granules and the resulting interstices can be seen with higher definition. This may be associated (ignoring the possible topographic effect) to lower polymer densities (higher free volume) of these interstices in support of the probable presence of slit-like pores within these gaps. AFM, at least directly, is not able to determine pore sizes in membranes having this structure.

From the analysis of our images, we could get slit pore sizes of $6.7 \pm 1.8 \mathrm{~nm}$ in thickness and lengths of $42 \pm 12 \mathrm{~nm}$. These sizes are bigger than those that would be expected in a NF membrane. This could be explained because these sizes correspond to the entrance of the pore formed by the granules that would be narrowed inside the membrane, and because they were actually observed with a tip larger than the pore. Really, the size 
visualized in the image depends on the curvature and the size of the granules and on the tip geometry. The size observed is always larger than the real size of the pores. Figure 5.b schematizes the difference between the real profile of the surface and that provided by the tip, with the consequent increase of the estimated pore size. However, AFM analysis clearly confirms that the pores of our membrane should have a more slit-like section.

Some more accurate morphological characteristics including the mean pore radius, $r_{\mathrm{p}}$, and the active layer thickness, $\Delta x_{a}$, must be known to be used as inputs for the model resolution presented above. A mean pore radius of $0.46 \pm 0.08 \mathrm{~nm}$ was obtained supposing slit pores in retention measurement. In the experimental measurements, tetraethylene glycol was used, taking concentration polarization into account and following a procedure exposed elsewhere by us [20] in order to obtain the true retention coefficient and the corresponding pore-size distribution. This result is close to the $0.48 \mathrm{~nm}$ obtained for this membrane by Hussain et al. [23] using uncharged solute rejection measurements and has been used in previous works $[10,20,21]$. The validity of the neutral solute retention method to get information on pore size has been previously tested by us [38]. The thickness of the active layer, $\Delta x_{a}$, was measured in our previous work [10] by Environmental Scanning Electron Microscopy images of transversal sections $\left(\Delta x_{a}=90 \pm 30 \mathrm{~nm}\right)$.

The transport number of ions inside the membrane was determined from measurements of membrane potential. Figure 6 shows membrane potential versus the logarithm of the concentrations ratio. The transport number of the $\mathrm{Na}^{+}$cation inside the pores of the active layer, $t_{1, p}$, can be determined from the slope of the straight fitted according to [50]:

$$
E_{\text {memb }}=\left(1-2 t_{1, p}\right) \frac{R T}{F} \ln \left(\frac{c_{\text {high }}}{c_{\text {low }}}\right)
$$


where $R$ is the gas constant, $T$ the absolute temperature and $F$ the Faraday constant. In

bigger than the free solution one: $t_{1, \mathrm{~b}}=0.39 \pm 0.01$ [51]. This means that there is a clear increase in the portion of transport carried by cations through the pores. The isoelectric point for this membrane is less than 3.3, as found in literature [52]. This means that the membrane is negatively charged when working with these ionic solutions.

The value obtained for water permeability was: $L_{\mathrm{w}}=(2.78 \pm 0.05) \cdot 10^{-11} \mathrm{~m} / \mathrm{s} \cdot \mathrm{Pa}$. From Equation (4) and taking $\eta_{\mathrm{b}}=8 \cdot 9 \cdot 10^{-4} \mathrm{~Pa} \cdot \mathrm{s}$ for the bulk viscosity [53], the pore viscosity $\eta_{\mathrm{pslit}}$ obtained is $(5.4 \pm 0.6) \cdot 10^{-3} \mathrm{~Pa} \cdot \mathrm{s}$. And with Equation (3) we can obtain the value of the porosity- thickness ratio: $(4.7 \pm 0.8) \cdot 10^{-7} \mathrm{~m}$. All the membrane properties are summarized in Table 1. 
Table1: Modeling fixed parameters.

$L_{\mathrm{w}}$

$\varepsilon_{\mathrm{d}}$

$\Delta x_{\mathrm{a}}$

$t_{1, \mathrm{p}}$

$r_{\mathrm{p}}$

$\eta_{\mathrm{p} s i t}$

$\Delta x_{\mathrm{a}} / A_{\text {ka }}$

$A_{\mathrm{ka}}$
$(2.78 \pm 0.05) \cdot 10^{-11} \mathrm{~m} / \mathrm{s} \cdot \mathrm{Pa}$

3

$90 \pm 30 \mathrm{~nm}$

$0.72 \pm 0.03$

$0.46 \pm 0.08 \mathrm{~nm}$

$(5.4 \pm 0.6) \cdot 10^{-3} \mathrm{~Pa} \cdot \mathrm{s}$

$(4.7 \pm 0.8) \cdot 10^{-7} \mathrm{~m}$

$0.19 \pm 0.09$

\subsection{Impedance Spectroscopy results and modeling}

Results obtained with impedance spectroscopy for $\mathrm{NaCl}$ solutions for this membrane were shown previously [21]. The Nyquist's plot has a very similar behavior to the results obtained for $\mathrm{KCl}$ solutions with the same concentration values [10]. An example of a Nyquist plot for the studied concentrations is shown in Figure 7. The first lobe, corresponding to high frequency (low real impedance) represents the solution outside the membrane flowing through the cell and inside the membrane support where there are no restrictions. The second lobe (lower frequency) is attributable to the really restrictive part of the membrane; i.e. to the

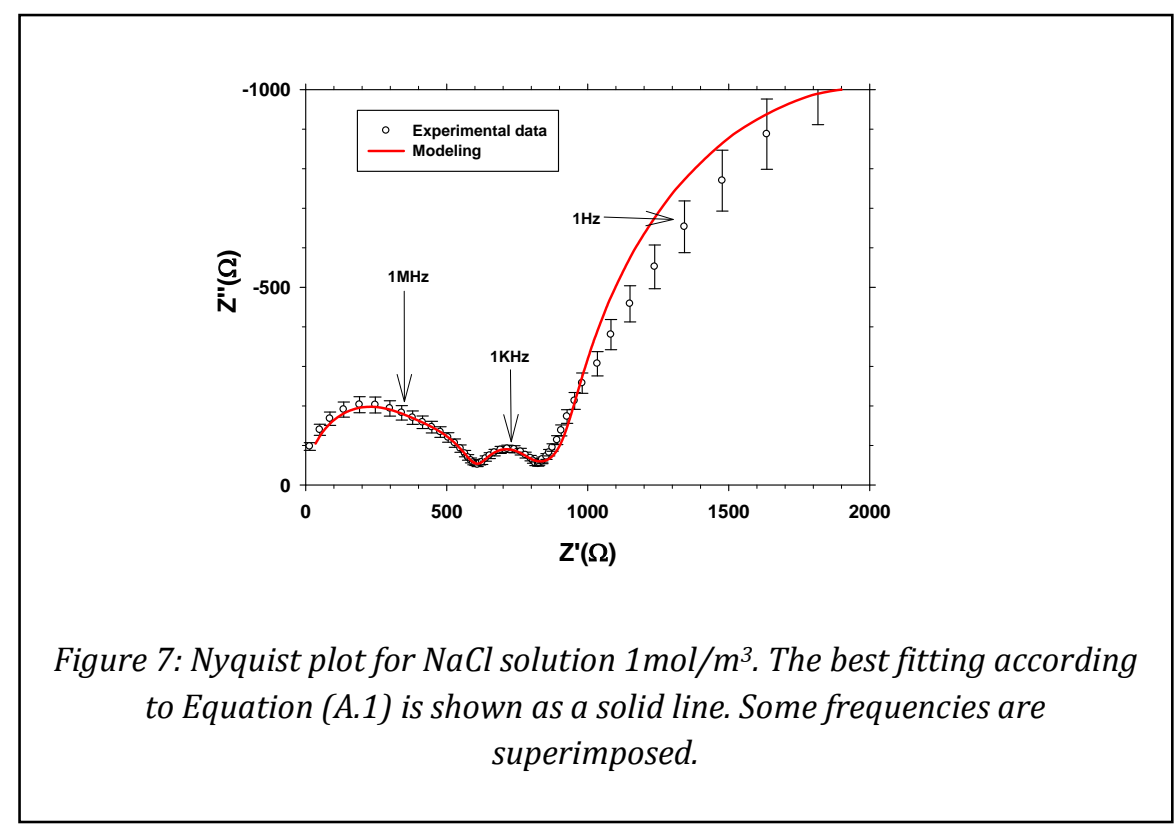


pores of the active layer of the membrane. The last lobe, for the lowest frequencies (high real impedances) corresponds to the relaxation of the polarization layer in contact with the electrodes and membrane (see Figure 1). The second lobe only appears when the membrane is present [10], (see supplementary Figure, S-1). It can be seen that the central lobe is where experimental data best fit the model described by Equation (A.1).

Following the procedure outlined in section 2.1, dielectric parameters of our system are obtained. In Table 2 the conductance and capacitance of the active layer of the membrane are shown, for $\mathrm{NaCl}$ concentrations studied.

Table 2: Capacities and conductances of the active layer of the membrane.

\begin{tabular}{lccccccc}
\hline & \multicolumn{7}{c}{ Concentration $\left(\mathrm{mol} / \mathrm{m}^{3}\right)$} \\
\cline { 2 - 8 } & 0.01 & 0.02 & 0.1 & 0.2 & 1 & 2 & 10 \\
\hline$C_{\text {memb }}(\mathrm{nF})$ & $990 \pm 30$ & $990 \pm 30$ & $790 \pm 20$ & $700 \pm 20$ & $600 \pm 18$ & $610 \pm 18$ & $610 \pm 18$ \\
$\mathrm{G}_{\text {memb }}(\mu \mathrm{S})$ & $345 \pm 10$ & $374 \pm 11$ & $513 \pm 15$ & $840 \pm 30$ & $4590 \pm 160$ & $9600 \pm 300$ & $49700 \pm 1500$ \\
\hline
\end{tabular}

\subsection{Permittivity and conductivity inside NF pores}

The permittivity inside the pores, $\varepsilon_{\mathrm{p}}$, can be obtained from capacities (table 2) and Equation (1). In this equation, a value of 3.0 has been assumed for the relative permittivity of the dry polymer (polyamide), $\varepsilon_{\mathrm{d}},[54-55]$. In Figure 8.a, the permittivity inside the pores, $\varepsilon_{p}$, is plotted against the concentration for slit geometries and also the wet membrane permittivity is presented, $\varepsilon_{\text {memb }}$ (according Equation (A.8)). The membrane permittivity is obviously lower than that of the pores. Solid lines in Figure 8.a correspond to the fittings to a three parameter exponential decay approximation, which was later used for the extrapolation of $\varepsilon_{p}$ values at higher concentrations.

The dependence of permittivity with membrane thickness is shown by including two dashed curves representing the variations in $\varepsilon_{\mathrm{p}}$ due to the variations in the estimation of $\Delta x_{\mathrm{a}}$ of $\pm 30 \mathrm{~nm}$. 

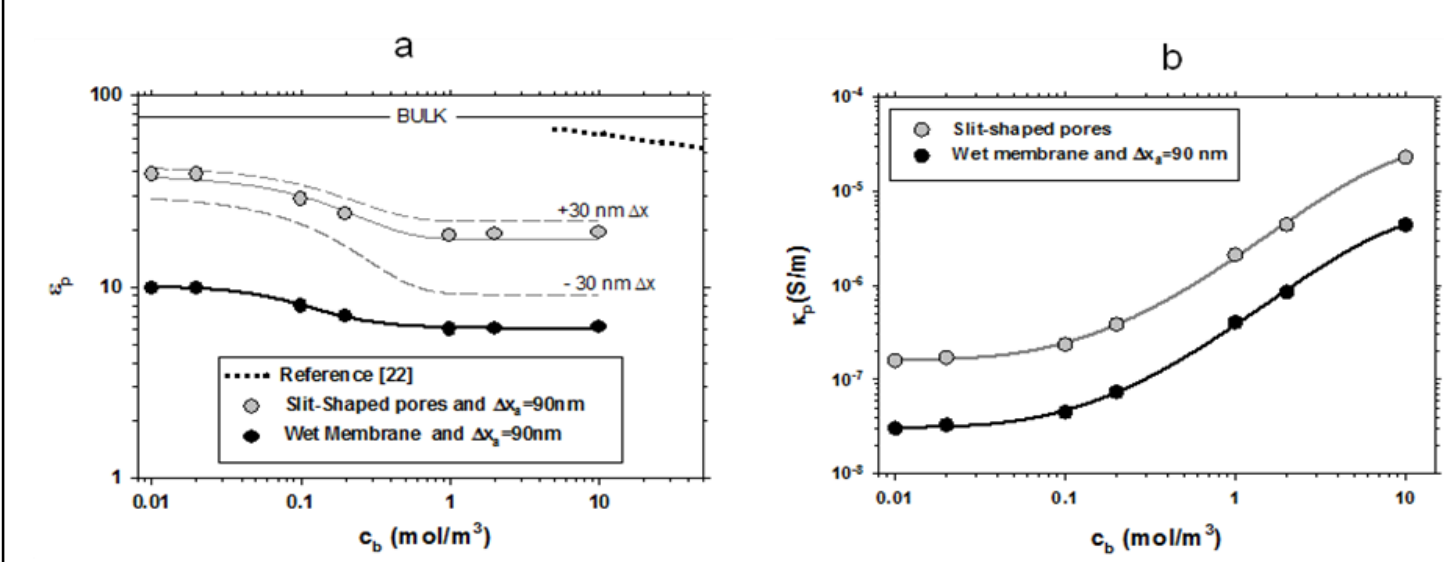

Figure 8: a) Permittivity inside membrane pores along with the global one for the wet membrane as a function of concentration. The dependence of permittivity on the membrane thickness is also included by showing the $\pm 30 \mathrm{~nm}$ dashed lines. For the wet membrane, the corresponding lines have not been drawn to avoid unnecessary complications in the figure. The results for $\varepsilon_{p}$ inside the pores obtained by fitting the SEDE-VCh model are also shown as a dotted line. b) Conductivity inside the pores and for the wet membrane, as function of concentration.

The results for permittivity inside the pores obtained from the flow and retention data for $\mathrm{NaCl}$ solutions, fitted to the SEDE-VCh model in a previous work [22] is also included in Figure 8.a versus the concentration, as a dotted line. In this case, the free parameters in the fit were the permittivity inside the pore and the two Freundlich isotherm constants. The discrepancy in the permittivity values inside the pores obtained by impedance spectroscopy and from retention data confirms the possibility that different pairs of values $\left(X, \varepsilon_{\mathrm{p}}\right)$ give a good fit to the data retention as demonstrated by Lanteri et al.[1].

The $\varepsilon_{\mathrm{p}}$ values obtained here for $\mathrm{NaCl}$ solutions are very similar to those obtained for $\mathrm{KCl}$ and the same membrane in a previous work [10]. The differences between the two salts are well within the experimental error. This has also been recently confirmed by other authors [56] for other membrane and salts. It seems that the changes in $\varepsilon_{p}$ are due to the confinement effects and to the concentration more than to the type of salt, at least for simple salts of a $1: 1$ type. 
The conductivity inside the pores is calculated with conductance values (table 2) and Equation (2). We assume that the polymer conductivity is much lower than inside the solution filled pores [57]. Thus, the second term on the right of Equation (2) can be neglected. Taking into account Equation (A.10), the conductivity inside the pores is:

$$
\kappa_{\mathrm{p}}=G_{\text {memb }}\left(\frac{\Delta x_{\mathrm{a}}}{A_{\mathrm{ka}} S}\right)
$$

Note, that in this case it is not necessary to know the thickness of the active layer because the thickness to porosity ratio is obtained directly from the measurements of water permeability by using Equation (3).

Figure $8 . \mathrm{b}$ compares the conductivity into the pores with that of the active layer of the membrane, which is almost an order of magnitude higher. This value is reasonable taking into account that the porosity of the active layer, for slit pores, is only $19 \%$. The conductivity inside the pores is more than three orders of magnitude lower than in free solution (not presented in Figure $8 . \mathrm{b}$ but in the $2.2 \cdot 10^{-4} \mathrm{~S} / \mathrm{m}$ to $1.2 \cdot 10^{-1}$ range. This fact corresponds to an entirely predictable effect of confinement into the pores reducing ionic mobility.

\subsection{Volumetric charge density and ionic mobility inside NF pores}

However, in Figure 9 it can be seen, that the ratio of pore to bulk conductivity varies only slightly, and fits fairly well to Equation (9), for bulk concentrations between 0.01 and $10 \mathrm{~mol} / \mathrm{m}^{3}$. Here $\Delta x_{\mathrm{a}}=90 \mathrm{~nm}$ has been assumed.

For the fitting of Equation (9), the transport number obtained from the membrane potential measurement has been used. Because the transport numbers are essentially independent of concentration the ratio of mobilities, $U_{1, \mathrm{p}} / U_{1, \mathrm{~b}}$, should also be almost concentration-independent. Then, the results on $\kappa_{\mathrm{p}} / \kappa_{\mathrm{b}}$ as a function of bulk concentration for 
Table 3: Mobilities ratio and Freundlich isotherm parameters as a function of active layer thickness.

\begin{tabular}{cccc}
\hline$\Delta x_{\mathrm{a}}(\mathrm{nm})$ & $\left(U_{1, \mathrm{p}} / U_{1, \mathrm{~b}}\right) \cdot 10^{2}$ & $\gamma \cdot 10^{3}\left(\mathrm{~mol} / \mathrm{m}^{3}\right)$ & $\Gamma$ \\
\hline 120 & $10.3 \pm 0.2$ & $9.10 \pm 0.05$ & $1.02 \pm 0.06$ \\
\hline 90 & $2.43 \pm 0.08$ & $29.3 \pm 0.1$ & $0.870 \pm 0.009$ \\
\hline 60 & $1.48 \pm 0.06$ & $30.9 \pm 0.1$ & $0.738 \pm 0.008$ \\
\hline
\end{tabular}


Figure 10 shows the absorbed charge inside the pores of the membrane as a function of $\mathrm{NaCl}$ concentration. The results obtained by using the model proposed by $\mathrm{Li}$ and Zhao, where dielectric effects were not considered [58] are also shown. It is seen that, if these effects are not taken into account, the model overestimates the membrane charge, because it has to give the barrier effects on ionic mobilities otherwise contributed by the dielectric effects.

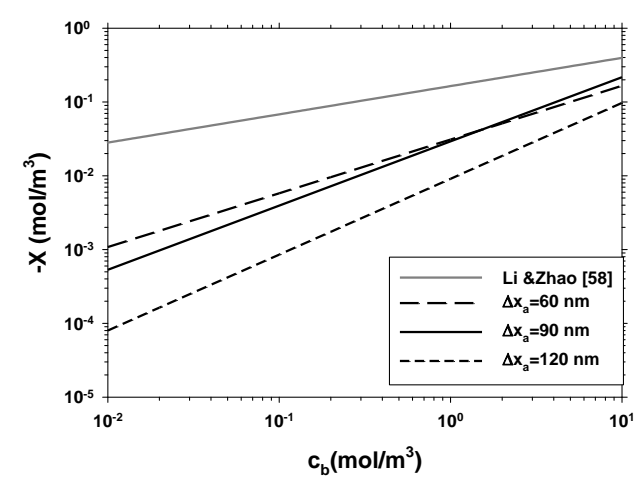

Figure 10: Freundlich isotherms for the volume charge density in the membrane as function of concentration.

\subsection{SEDE-VCh model predictions}

In order to obtain predictions on $\mathrm{NaCl}$ rejection, the isotherm presented in Figure 10 and the $\varepsilon_{\mathrm{p}}$ correlation in Figure 8.a were used as input of SEDE-VCh model (see appendix B (Table B.1)). As already mentioned in section 3.3, the rejection experimental values and the intrinsic rejection were taken from a previous work [22]. Both, theoretical and experimental results, are shown in Figure 11 as a function of the flux of permeate.

In this figure is can be seen that the goodness of the prediction decreases when concentration increases. There are several reasons for this behavior; firstly, the SEDE-VCh model should be applied to solutions relatively well diluted, where this assumption is still applicable. On the other side, the values of $\varepsilon_{\mathrm{p}}, \gamma$ and $\Gamma$, used for the highest concentration, are the result of extrapolation, which probably indicates that these values can be slightly 


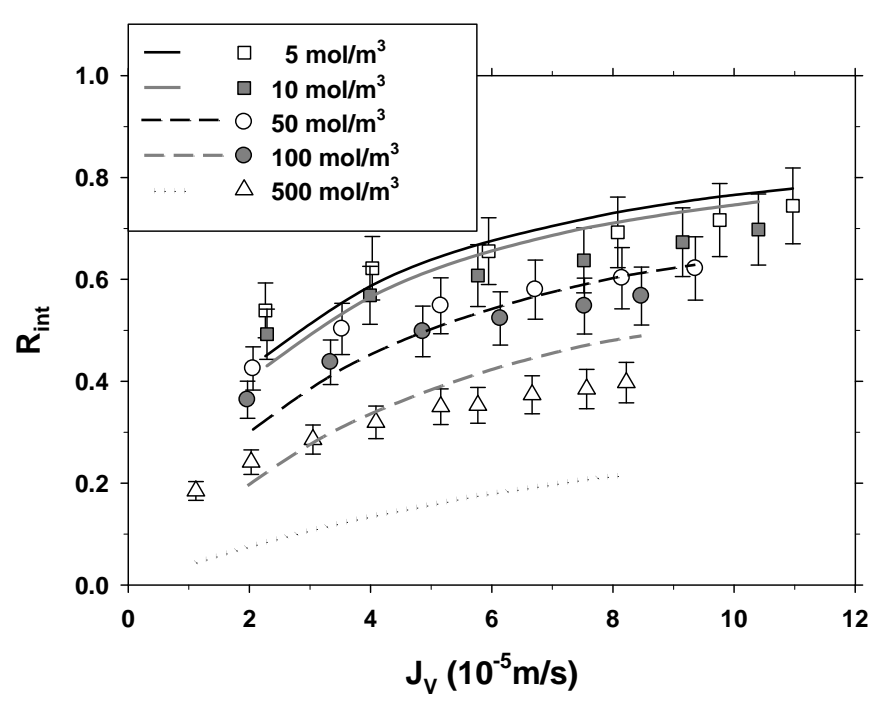

Figure 11: Intrinsic retention as a function of permeate flux for different feed concentrations: Experimental data (symbols) and model predicted values (lines).

different. Actually the extrapolation of the charge density ( $Y$ and $\Gamma$ values) for wide concentration ranges could be justified since it is based on the Freundlich model of heterogeneous adsorption, very commonly used to explain the equilibrium of ionic solutions with polymer surfaces, although high concentrations could introduce adsorbate-adsorbate interactions that would be not included within the Freundlich adsorption mechanism [31]. Moreover, $\varepsilon_{\mathrm{p}}$ is extrapolated according to the phenomenological curve (three parameter exponential decay) fitted to the experimental data (Figure 8.a) that can lose accuracy out of the range where it was evaluated.

In order to compare the accuracy of the prediction, Figure 12 shows the deviation in percentage between intrinsic retention values predicted by the model and experimental ones, as a function of feed concentration, defined as:

$$
D=\frac{1}{n} \sum_{j=1}^{n} \frac{R_{\mathrm{int}, j}(\exp )-R_{\mathrm{int}, j}(\mathrm{cal})}{R_{\mathrm{int}, j}(\exp )}
$$


Here $R_{\mathrm{in}, j}(\exp )$ is the intrinsic rejection evaluated from the experimental observed retention for each $J_{V, j}(\exp )$. $R_{\mathrm{int}, j}(\mathrm{cal})$ is the intrinsic retention predicted by the model and $\mathrm{n}$ is the number of values evaluated for each concentration. It can be seen that the concentration range where there is not any data extrapolation (concentrations lower than $10 \mathrm{~mol} / \mathrm{m}^{3}$, dark shaded area in Figure 12) the deviations are less than 3\%. For extrapolated concentrations, until 50 $\mathrm{mol} / \mathrm{m}^{3}$ (light shaded area in Figure 12), deviations are less than $5 \%$, whereas for higher concentrations, the model underestimates the retention values with higher deviations. As we have already mentioned, the main cause of this deviation should be the phenomenological extrapolation of permittivity, conductivity and transport numbers, however other factors such as the concept of dilute solutions in the model must also influence this discrepancy.

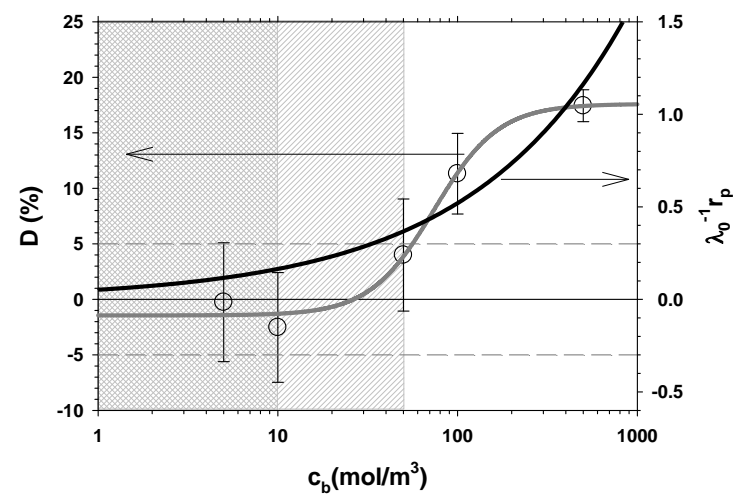

Figure 12: Left axis: Deviation in percentage between experimental retention values and intrinsic ones predicted by the model versus the concentration. The line is only an eye guide. Right axis: Product of the inverse Debye length and the pore radius versus concentration.

The method used in this study has some difficulty to determine accurately the permittivity, for concentrations above $10 \mathrm{~mol} / \mathrm{m}^{3}$, since the second lobe of the Nyquist plot (see Figure 7) is too small leading to the necessity of extrapolating over this concentration. However, recently Efligenir and co-workers [56] designed an experimental method to determine the permittivity by impedance spectroscopy, isolating the active layer of the 
membrane. Possibly, this technique could provide values of permittivity at higher concentrations for NF membranes.

In any case, to predict retention with high and moderately high concentrations, not only the activity coefficients in Equation (5), should be considered but also the influence of shielding, which increases with concentration, as pointed out by Dukhin et al. [29]. According to these authors, for membranes with moderately high charge, the simultaneous dielectric and charge mechanisms produce not simple multiplication of the Donnan and dielectric factor but a quadratic increase in the intensity of dielectric exclusion. It seems instructive to plot ratio of the pore radius to the Debye`s lengths, $r_{p} / \lambda_{D}$, that represent somehow the charge density. On the right axis of Figure 12 the evolution of $r_{p} / \lambda_{D}$ as a function of concentration is shown. It can be seen that the region where the deviation between retentions is higher than $5 \%$, correspond to $r_{\mathrm{p}} / \lambda_{\mathrm{D}} \geq 0.3$. If for high values of concentration, Equation (5) were changed by introducing this quadratic dependence of dielectric exclusion energy, the retention values calculated would be higher, and probably would approach to the experimental data.

Of course some aspects of the model itself could be improved. For instance the parallel pore approximation, used to calculate the dielectric constant inside the pore, that assumes that the pores are an array of homogeneous equally sized slits, could be improved by an adequate, but difficult to assess, distribution of pore sizes on the surface and along the pores themselves. Nevertheless as a consequence of the dielectric behavior of porous walls, the overall dielectric permeability and conductivity are more trustable than the actual pore size and thickness values. Other possible source of errors could be due to the assumption of a independence and separability of the effects of active and support layers that although tested by us could not be total and especially for high concentrations. 
Another quite important expansion of the work presented here could be in the direction of including multi-ionic salts. Actually, the knowledge of competitive adsorption isotherms in systems containing several anions remains the main challenge for assessing the predictive abilities of the methodology proposed, although the transport modeling is relatively easy to generalize [22]. Of course in such cases impedance spectroscopy and membrane potential contributions to the methodology studied here have to be modified, and are being improved, too."

\section{Conclusions}

From measurements of impedance spectroscopy and applying the model of interfacial equilibrium we can conclude that: The Impedance Spectroscopy method allows a separation of the different relaxation processes appearing in a complex membrane system; although certainly, it is worth taking into account that some aspects of the model could be still improved as mentioned. Experimental results can be fitted to obtain the conductivity and permittivity inside the membrane pores, and by modeling, the charge inside the pores. The confinement of the ions inside the pores reduces both the ionic mobility and the relative permittivity. The increase in concentration only slightly reduces the value of the permittivity inside the pores, whereas the ion mobility varies similarly to that of the ions in the free solution.

Regarding the application of SEDE-VCh model, we can say that, obtaining the model parameters by independent methods, the model can be used in a predictive way for NF process. The results are good enough considering the complexity of the mechanisms involved. The best prediction was found for the diluted concentration according with the assumption of the model and the area where the parameters used have been calculated. 


\section{Appendix A}

The electric and dielectric characteristics can be obtained, by means of the conductance $G$ and capacitance $C$. The whole system can be modelled by an extension of the Maxwell-Wagner theory, in which the overall complex capacitance is obtained by a method similar to that used by Kita [59] and Asami [60] according to our adaptation [10] and as

$$
C^{*}(\omega)=C_{N, \mathrm{~h}}+\sum_{i=1}^{N-1} \frac{\left(C_{i, 1}-C_{i, \mathrm{~h}}\right)}{1+\left(j \omega \tau_{i}\right)^{1-\alpha_{i}}}-j \frac{G_{1,1}}{\omega}
$$

Where $N$ is the total number of phases, $\tau_{i}=(1 / 2) \pi f_{i}$ is the relaxation time and $f_{i}$ is the frequency at the maximum value in the Nyquist plot (the complex plane plot of impedances) for the i-th phase, $\omega$ is the angular frequency, $\omega=2 \pi f$ (of course also $\left.\omega_{i}=2 \pi f_{i}\right), C_{N, \mathrm{~h}}$ is the capacitance at infinite frequency, $C_{i, 1}$ and $C_{i, \mathrm{~h}}$ are capacitances for each relaxation time, at low and high frequencies respectively, and $G_{1,1}$ is the conductance of the system at low frequency. The $\alpha_{i}$ parameters are distribution factors characterizing the spread of relaxation times [60]. $\alpha_{i}$ is 0 , or very near to 0 , when the process has a single relaxation time (Debye type) and the corresponding curve in the Nyquist plot is a perfect semicircle with its center over the real axis. When the curve presents deviations from a semicircle, the value of $\alpha_{i}$ is nonzero.

For multilayer systems some constraints in Eq. (A.1) must be fulfilled. These are:

$$
\begin{aligned}
& C_{i, \mathrm{~h}}=C_{(i+1), 1} \quad \text { for } \quad i=1, \ldots, N-1 \\
& G_{i, \mathrm{~h}}=G_{(i+1), 1} \quad \text { for } \quad i=1, \ldots, N-1
\end{aligned}
$$

Using experimental results (left side in Eq. (A.1)) it is possible to identify the dielectric parameters $G_{i, \mathrm{~h}}, G_{1,1}, C_{N, \mathrm{~h}}, C_{i, h}$ and $\alpha_{i}$ for all i. The relation between these 
parameters and those of the layers was presented by Li and Zhao [58] and confirmed by us [10]. This description is similar to assuming an equivalent circuit of a number of elements formed by the combination of a capacitance and a conductance in parallel. In the frequency range corresponding to the time relaxation of the solution inside the membrane, the phase parameters can be obtained according to the equations presented in Table A.1. In particular, permittivity and conductivity in the active layer of the membrane are given by Equations (A.8) and (A.10) in Table A.1 respectively.

Table A-1. Relation between phase and dielectric parameters [58].

$$
\begin{aligned}
& \omega=\frac{G_{\mathrm{h}}-G_{1}}{C_{1}-C_{h}}=2 \pi f \\
& B=\omega+\frac{G_{h}}{C_{h}} \\
& D=\sqrt{B^{2}-4 \frac{G_{1} \omega}{C_{h}}} \\
& A=\frac{B-D}{2} \\
& C_{\text {memb }}=C_{\mathrm{h}} \frac{D}{\omega-A} \\
& C_{\mathrm{b}}=C_{\mathrm{h}} \frac{D}{b-\omega} \\
& \varepsilon_{\mathrm{b}}=C_{\mathrm{b}} \frac{L}{S \varepsilon_{0}} \\
& G_{\text {memb }}=A C_{\text {memb }} \\
& \kappa_{\text {memb }}=G_{\text {memb }} \frac{\Delta x_{\mathrm{a}}}{S} \\
& G_{\mathrm{b}}=b C_{\mathrm{b}} \\
& \kappa_{\mathrm{b}}=G_{\mathrm{b}} \frac{L}{S}
\end{aligned}
$$

The sub-indexes $\mathrm{h}$ and $\mathrm{l}$ refer to the high and low frequencies and memb and $\mathrm{b}$ refer to inside and outside of each membrane system layer. 


\section{Appendix B}

In Table B.1 the relationships used within the SEDE-VCh model are shown. These equations include: transport equations inside the slit shaper pores, partitions at interfaces, dielectric energies and intrinsic retention, are shown.

Table B.1: SEDE-VCh equations for slit shaped pores.

\section{TRANSPORT EQUATIONS INSIDE PORES}

$$
\frac{d c_{i}}{d x}=\frac{J_{V}}{K_{i, \mathrm{~d}} D_{i, \infty} A_{\mathrm{ka}}}\left(K_{i, c}^{\prime} c_{i}-c_{i, \mathrm{perm}}\right)-\frac{z_{i} F}{R T} \frac{d \psi}{d x}
$$

$\sum_{i} c_{i} z_{i}+{ }_{c_{2}}{ }^{\Gamma}=0$

$\frac{d \psi}{d x}=\frac{\sum_{i}\left(\frac{z_{i} J_{V}}{K_{i, \mathrm{~d}} D_{i, \infty} A_{\mathrm{ka}}}\right)\left(K_{i, c}^{\prime} c_{i}-c_{i, \mathrm{perm}}\right)+\left(\frac{J_{V} \Gamma{ }_{c_{2}}{ }^{\Gamma-1}}{K_{i, \mathrm{~d}} D_{2, \infty} A_{\mathrm{ka}}}\right)\left(K_{2, c}^{\prime} c_{2}-c_{2, \mathrm{perm}}\right)}{\frac{F}{R T}\left(\sum_{i} c_{i} z_{i}^{2}+z_{2} Y_{c_{2}}{ }^{\Gamma}\right)}$

Boundary conditions

$$
\begin{aligned}
& c_{i}(x=0)=c_{i, 0} \\
& c_{i}(x=\Delta x)=c_{i, \Delta x_{a}}
\end{aligned}
$$

$$
K_{i, \mathrm{~d}}=\frac{1+\frac{9}{16} \lambda_{i} \ln \lambda_{i}-1.19358 \lambda_{i}+0.4285 \lambda_{i}^{3}-0.3192 \lambda_{i}^{4}+0.08428 \lambda_{i}^{5}}{1-\lambda_{i}}
$$

$$
K_{i, \mathrm{c}}=\frac{1-3.02 \lambda_{i}{ }^{2}+5.776 \lambda_{i}^{3}-12.3675 \lambda_{i}^{4}+18.9975 \lambda_{i}^{5}-15.2185 \lambda_{i}^{6}+4.8525 \lambda_{i}^{7}}{1-\lambda_{i}}
$$

$$
K_{i, \mathrm{c}}^{\prime}=K_{i, \mathrm{c}}+\left(2-\phi_{i}\right) K_{i, \mathrm{~d}}\left(\frac{2 \lambda_{i}}{3}\right)
$$

\section{PARTITIONING EQUATIONS}

$$
\frac{c_{i, 0}}{c_{i, m}}=\theta_{i} \exp \left(-z_{i} \Delta \Psi\right)(m \square 0) \text { and } \frac{c_{i, \Delta x_{a}}}{c_{i, p}}=\theta_{i} \exp \left(-z_{i} \Delta \Psi\right)\left(\text { perm } \square \Delta x_{a}\right)
$$

$(m \square 0)$ and $\left(\right.$ perm $\left.\square \Delta x_{a}\right)$ correspond to the feed side and permeate side, respectively, of the active layer of the membrane.

\section{THE DIELECTRIC BORN ENERGY}

$$
\Delta W_{i, \text { Borm }}^{\prime}=\frac{\left(z_{i} e\right)^{2}}{8 \pi k_{B} T \varepsilon_{0} a_{s}}\left(\frac{1}{\varepsilon_{\mathrm{p}}}-\frac{1}{\varepsilon_{\mathrm{b}}}\right)
$$


Where $a_{s}$ is the cavity radius defined by [61] as the distance from the center of the ion to the point where the relative permittivity becomes different than the vacuum one, $\varepsilon_{0}$.

\section{THE DIELECTRIC IMAGE FORCE ENERGY}

$$
\begin{aligned}
& \Delta W_{i, \text { im }}^{\prime}=-\frac{r_{\mathrm{B}}}{r_{\mathrm{p}}} \ln \left[1-\left(\frac{\varepsilon_{\mathrm{p}}-\varepsilon_{\mathrm{d}}}{\varepsilon_{\mathrm{p}}+\varepsilon_{\mathrm{d}}}\right) \exp (-2 \mu)\right] \\
& r_{B}=\frac{\left(z_{i} F\right)^{2}}{8 \pi \varepsilon_{0} \varepsilon_{\mathrm{p}} R T N_{\mathrm{A}}} \\
& \mu=\frac{r_{p}}{\lambda_{0}} \sqrt{\frac{I_{\mathrm{b}}}{I_{\mathrm{p}}}}
\end{aligned}
$$

Where $r_{B}$ is the Bjerrum radius, $\lambda_{0}$ is the Debye length. $I_{\mathrm{b}}$ and $I_{\mathrm{p}}$ are the ionic strength outside and inside the pores respectively.

\section{THE INTRINSIC RETENTION}

$$
R_{i, \text { int }}=1-\frac{c_{i, \text { perm }}}{c_{i, \mathrm{~m}}}
$$

\section{Acknowledgements}

Authors thank the Ministerio de Educación y Ciencia (Plan Nacional de I+D+i) through projects CTQ2012-31076 and MAT2011-25513 and Junta de Castilla y León (project VA248U13). 


\section{References}

[1] Y. Lanteri, P. Fievet, A. Szymczyk, Evaluation of the steric, electric, and dielectric exclusion model on the basis of salt rejection rate and membrane potential measurements, J. Colloid Interf. Sci., 331 (2009) 148-155.

[2] O. Kedem, A. Katchalsky, Thermodynamic analysis of the permeability of biological membranes to non-electrolytes, Biochim. Biophys. Acta, 27 (1958) 229-246.

[3] K.S. Spiegler, O. Kedem, Thermodynamics of hyperfiltration (reverse osmosis): criteria for efficient membranes, Desalination, 1 (1966) 311-326.

[4] J.D. Ferry, Statistical evaluation of sieve constants in ultratiltration, J. Gen. Physiol., 20 (1936) 95-104.

[5] S.-I. Nakao, S. Kimura, Models of Membrane-Transport Phenomena and Their Applications for Ultrafiltration Data, J. Chem. Eng. Jpn., 15 (1982) 200-205.

[6] K.H. Meyer, J.F. Sievers, La perméabilité des membranes I. Théorie de la perméabilité ionique, Helv. Chim. Acta, 19 (1936) 649-664.

[7] T. Teorell, Transport processes and electrical phenomena in ionic membranes, Prog. Biophys. Biophysical Chem., 3 (1953) 305-369.

[8] X.-L. Wang, T. Tsuru, S.-i. Nakao, S. Kimura, Electrolyte transport through nanofiltration membranes by the space-charge model and the comparison with TeorellMeyer-Sievers model, J. Membr. Sci., 103 (1995) 117-133.

[9] W.R. Bowen, A.W. Mohammad, N. Hilal, Characterisation of nanofiltration membranes for predictive purposes - use of salts, uncharged solutes and atomic force microscopy, J. Membr. Sci., 126 (1997) 91-105.

[10] M. Montalvillo, V. Silva, L. Palacio, J.I. Calvo, F.J. Carmona, A. Hernández, P. Prádanos, Charge and dielectric characterization of nanofiltration membranes by impedance spectroscopy, J. Membr. Sci., 454 (2014) 163-173. 
[11] D. Vezzani, S. Bandini, Donnan equilibrium and dielectric exclusion for

characterization of nanofiltration membranes, Desalination, 149 (2002) 477-483.

[12] W.R. Bowen, H. Mukhtar, Characterisation and prediction of separation performance of nanofiltration membranes, J. Membr. Sci., 112 (1996) 263-274. [13] W.R. Bowen, A.W. Mohammad, Characterization and Prediction of Nanofiltration Membrane Performance-A General Assessment, Chem. Eng. Res. Des., 76 (1998) 885893.

[14] S. Bandini, D. Vezzani, Nanofiltration modeling: the role of dielectric exclusion in membrane characterization, Chem. Eng. Sci., 58 (2003) 3303-3326.

[15] A. Szymczyk, P. Fievet, Investigating transport properties of nanofiltration membranes by means of a steric, electric and dielectric exclusion model, J. Membr. Sci., 252 (2005) 77-88.

[16] S. Déon, A. Escoda, P. Fievet, R. Salut, Prediction of single salt rejection by NF membranes: An experimental methodology to assess physical parameters from membrane and streaming potentials, Desalination, 315 (2013) 37-45. [17] V. Silva, Á. Martín, F. Martínez, J. Malfeito, P. Prádanos, L. Palacio, A. Hernández, Electrical characterization of NF membranes. A modified model with charge variation along the pores, Chem. Eng. Sci., 66 (2011) 2898-2911. [18] C.Y. Tang, Y.-N. Kwon, J.O. Leckie, Effect of membrane chemistry and coating layer on physiochemical properties of thin film composite polyamide RO and NF membranes: I. FTIR and XPS characterization of polyamide and coating layer chemistry, Desalination, 242 (2009) 149-167.

[19] C.Y. Tang, Y.-N. Kwon, J.O. Leckie, Effect of membrane chemistry and coating layer on physiochemical properties of thin film composite polyamide RO and NF 
membranes: II. Membrane physiochemical properties and their dependence on polyamide and coating layers, Desalination, 242 (2009) 168-182.

[20] N. García-Martín, V. Silva, F.J. Carmona, L. Palacio, A. Hernández, P. Prádanos, Pore size analysis from retention of neutral solutes through nanofiltration membranes. The contribution of concentration-polarization, Desalination, 344 (2014) 1-11.

[21] M. Montalvillo, V. Silva, L. Palacio, A. Hernandez, P. Pradanos, Dielectric properties of electrolyte solutions in polymeric nanofiltration membranes, Desalin. Water Treat., 27 (2011) 25-30.

[22] V. Silva, V. Geraldes, A.M. Brites Alves, L. Palacio, P. Prádanos, A. Hernández, Multi-ionic nanofiltration of highly concentrated salt mixtures in the seawater range, Desalination, 277 (2011) 29-39.

[23] A.A. Hussain, S.K. Nataraj, M.E.E. Abashar, I.S. Al-Mutaz, T.M. Aminabhavi, Prediction of physical properties of nanofiltration membranes using experiment and theoretical models, J. Membr. Sci., 310 (2008) 321-336.

[24] S. Van Geluwe, C. Vinckier, L. Braeken, B. Van der Bruggen, Ozone oxidation of nanofiltration concentrates alleviates membrane fouling in drinking water industry, $\mathrm{J}$. Membr. Sci., 378 (2011) 128-137.

[25] K. Wesolowska, S. Koter, M. Bodzek, Modelling of nanofiltration in softening water, Desalination, 163 (2004) 137-151.

[26] W.R. Bowen, J.S. Welfoot, Modelling the performance of membrane nanofiltration-critical assessment and model development, Chem. Eng. Sci., 57 (2002) 1121-1137. [27] A.E. Yaroshchuk, Non-steric mechanisms of nanofiltration: superposition of Donnan and dielectric exclusion, Sep. Purif. Technol., 22-23 (2001) 143-158.

[28] J.N. Israelachvili, Intermolecular and surface forces / Jacob N. Israelachvili, Academic Press, London ; San Diego, 1991. 
[29] S.S. Dukhin, N.V. Churaev, V.N. Shilov, V.M. Starov, Modelling Reverse Osmosis, Russ. Chem. Rev., 57 (1988) 572.

[30] A.E. Yaroshchuk, Dielectric exclusion of ions from membranes, Adv. Colloid Interfac. Sci., 85 (2000) 193-230.

[31] A.W. Adamson, Physical Chemistry of Surfaces, Wiley, New York, 1982.

[32] J.I. Calvo, A. Hernández, P. Prádanos, F. Tejerina, Charge Adsorption and Zeta Potential in Cyclopore Membranes, J. Colloid Interf. Sci., 181 (1996) 399-412.

[33] L. Dresner, Some remarks on the integration of the extended Nernst-Planck equations in the hyperfiltration of multicomponent solutions, Desalination, 10 (1972) 27 46.

[34] R. Schlögl, Membrane permeation in systems far from equilibrium, Ber. Bunsen. Phys. Chem., 70 (1966) 400-414.

[35] T. Tsuru, S.-i. Nakao, S. Kimura, Calculation of Ion Rejection by Extended NernstPlanck Equation with Charged Reverse Osmosis Membranes for Single and Mixed Electrolyte Solutions, J. Chem. Eng. Jpn., 24 (1991) $511-517$.

[36] F. Fadaei, V. Hoshyargar, S. Shirazian, S.N. Ashrafizadeh, Mass transfer simulation of ion separation by nanofiltration considering electrical and dielectrical effects, Desalination, 284 (2012) 316-323.

[37] Y. Cai, X. Chen, Y. Wang, M. Qiu, Y. Fan, Fabrication of palladium-titania nanofiltration membranes via a colloidal sol-gel process, Micropor. Mesopor. Mat., 201 (2015) 202-209.

[38] J.A. Otero, O. Mazarrasa, J. Villasante, V. Silva, P. Prádanos, J.I. Calvo, A. Hernández, Three independent ways to obtain information on pore size distributions of nanofiltration membranes, J. Membr. Sci., 309 (2008) 17-27. 
[39] S. Bouranene, P. Fievet, A. Szymczyk, Investigating nanofiltration of multi-ionic

solutions using the steric, electric and dielectric exclusion model, Chem. Eng. Sci., 64 (2009) 3789-3798.

[40] S. Déon, P. Dutournié, P. Bourseau, Modeling nanofiltration with Nernst-Planck approach and polarization layer, AIChE J., 53 (2007) 1952-1969.

[41] V. Silva, Theoretical foundations and modelling in nanofiltration membrane systems, in: Física Aplicada, Universidad de Valladolid, Valladolid, 2009, pp. 208. [42] V.M. Starov, N.V. Churaev, Separation of electrolyte solutions by reverse osmosis, Adv. Colloid Interfac. Sci., 43 (1993) 145-167.

[43] L. Dresner, Ion exclusion from neutral and slightly charged pores, Desalination, 15 (1974) 39-57.

[44] D. Johnson, N. Hilal, Characterisation and quantification of membrane surface properties using atomic force microscopy: A comprehensive review, Desalination, 356 (2015) 149-164.

[45] I. Schmitz, M. Schreiner, G. Friedbacher, M. Grasserbauer, Phase imaging as an extension to tapping mode AFM for the identification of material properties on humidity-sensitive surfaces, App. Surf. Sci., 115 (1997) 190-198.

[46] L. Martinez, M.A. Gigosos, A. Hernandez, F. Tejerina, Study of some electrokinetic phenomena in charged microcapillary porous membranes, J. Membr. Sci., 35 (1987) 120.

[47] G. Vazquez, E. Alvarez, J.M. Navaza, Surface Tension of Alcohol Water + Water from 20 to 50 .degree.C, J. Chem. Eng. Data, 40 (1995) 611-614.

[48] D.J. Johnson, S.A. Al Malek, B.A.M. Al-Rashdi, N. Hilal, Atomic force microscopy of nanofiltration membranes: Effect of imaging mode and environment, J. Membr. Sci., 389 (2012) 486-498. 
[49] J. Stawikowska, A.G. Livingston, Assessment of atomic force microscopy for characterisation of nanofiltration membranes, J. Membr. Sci., 425-426 (2013) 58-70.

[50] N. Lakshminarayanaiah, Transport phenomena in membranes, Academic Press, New York, 1969.

[51] R.A. Robinson, R.H. Stokes, Electrolyte Solutions: Second Revised Edition, Second Edition, Revised ed., Dover, London, 2002.

[52] P. Religa, A. Kowalik-Klimczak, P. Gierycz, Study on the behavior of nanofiltration membranes using for chromium(III) recovery from salt mixture solution, Desalination, 315 (2013) 115-123.

[53] D.R. Lide, CRC Handbook of Chemistry and Physics, 85 ed., CRC Press, Boca Raton, FL, 2005.

[54] M.O. Aboelfotoh, C. Feger, Frequency dependence of dielectric loss in thin aromatic polyimide films, Phys. Rev. B, 47 (1993) 13395-13400.

[55] W.-J. Shang, X.-L. Wang, Y.-X. Yu, Theoretical calculation on the membrane potential of charged porous membranes in 1-1, 1-2, 2-1 and 2-2 electrolyte solutions, J. Membr. Sci., 285 (2006) 362-375.

[56] A. Efligenir, P. Fievet, S. Déon, R. Salut, Characterization of the isolated active layer of a NF membrane by electrochemical impedance spectroscopy, J. Membr. Sci., 477 (2015) 172-182.

[57] H.-L. Shao, S. Umemoto, T. Kikutani, N. Okui, Electrical Conductivity in Nylon 66 Thin Films Prepared by Alternating Vapor Deposition Polymerization, Polym. J, 31 (1999) 1083-1088.

[58] Y.H. Li, K.S. Zhao, Dielectric analysis of nanofiltration membrane in electrolyte solutions: influences of electrolyte concentration and species on membrane permeation, J. Colloid Interf. Sci., 276 (2004) 68-76. 
[59] Y. Kita, Dielectric-Relaxation in Distributed Dielectric Layers, J. Appl. Phys., 55

(1984) 3747-3755.

[60] K. Asami, Characterization of heterogeneous systems by dielectric spectroscopy, Prog. Polym. Sci., 27 (2002) 1617-1659.

[61] A.A. Rashin, B. Honig, Reevaluation of the Born model of ion hydration, J. Phys. Chem., 89 (1985) 5588-5593.

Symbol lists

$A, b, B, D \quad$ Parameters defined in Table A.1

$A_{\mathrm{k}} \quad$ Porosity

$a_{s} \quad$ Cavity radius

C Capacitance

$C^{*} \quad$ Complex capacitance

c Concentration

d Thickness of the layer in Equation (4)

$E_{\text {cell }} \quad$ Cell potential

$E_{\text {memb }} \quad$ Membrane potential

$E_{\text {Nernst }} \quad$ Solution potential

$e \quad$ Elementary charge

F Faraday constant

f Frequency

G Conductance

$h \quad$ thickness of the slit 
Ionic strength

$J_{V}$

j

$K_{i, c}$

$K_{i, c}^{\prime}$

$K_{i, d}$

$k_{B}$

$L$

$L_{\mathrm{w}}$

$N$

$N_{\text {A }}$

$R$

$R_{\text {int }}$

$r_{\mathrm{p}}$

$r_{\text {Stokes }}$

$S$

$T$

$t$

$U$

$x$

$z$
Volumetric flux per unit of membrane area

Imaginary number $\sqrt{-1}$

Hindrance factor for convection

Hindrance factor for convection with pressure gradient effect

Hindrance factor for diffusion

Boltzmann constant

Thickness of layer

Water permeability

Layer number, pores number or $\mathrm{Cl}^{-}$adsorbed number.

Avogadro's number

Universal constant of gases

Intrinsic retention

Pore radius

Stokes radius

Membrane area

Temperature

Transport numbers

Mobility

Coordinate

Ion valence 


\section{Greeks letters}

$\alpha$

$X$

$\Delta W_{i, \text { Born }}^{\prime}$

$\Delta W_{i, \mathrm{im}}^{\prime}$

$\Delta x$

$\Delta \Psi \quad$ Normalized Donnan potential

$\varepsilon \quad$ Relative permittivity

$\varepsilon_{0}$

$\varepsilon_{\mathrm{w}}$

$\phi$

$\Gamma$

$\gamma$

$\eta$

$\kappa$

$\lambda$

$\lambda_{0}$

$\mu$

$\theta$

$\tau$

$\omega$

$\psi$

r

Volume charge density

Layer thickness

Vacuum permittivity

Steric coefficient

Parameter in Equation (8)

Activity coefficient

Viscosity

Conductivity

Debye length

Time relaxation

Angular frequency

Electric potential

Parameter in Equation (8)
Distribution factor of relaxation times

Free energy difference due to Born effects

Free energy difference due to images forces effects

Relative permittivity of the water

Ratio of ion radius to pore radius

Parameter defined in Equation (B.13)

Coefficient grouping the influence of steric and dielectric effects. 


\section{Sub index}

Just inside of membrane in the feed side

b

c

d

h

high

$i$

1

low

$\mathrm{m}$

memb

$\mathrm{p}$

perm

$\mathrm{S}$

$\Delta x_{\mathrm{a}}$
Active layer of the membrane.

Bulk

Convection in Equations (B.6) and (B.7)

Dry membrane or diffusion in Equation (B.5)

High frequency

Side of high concentrations in the membrane potential measurements

Number of relaxation time $i=1, \ldots, \mathrm{N}-1$ or ion $(1$ for the cation and 2 for the anion)

Low Frequency

Side of low concentrations in the membrane potential measurements

Just offside of membrane in the feed side

Membrane

Pore

Permeate or just offside of active layer of the membrane in the permeate side

Porous support

Just inside of active layer of the membrane in the permeate side. 


\section{Figure List}

Figure 1: Schematic representation of the impedance measurement system.

Figure 2: Scheme of concentration profile in the pores of the active layer of the membrane, a) on the experiences of Impedance Spectroscopy, b) on the experiences of NF of a solution.

Figure 3: Schematic representation of the calculation procedure.

Figure 4: AFM 500x500 nm image of DESAL-HL membrane: In air a) Topography b) Phase, and in ethanol c) topography d) phase.

Figure 5: Scheme of AFM analysis of the membrane Surface a) Phase change between hard and soft zones on the Surface b) Topography measure (difference between the real profile of the Surface and the obtained one)

Figure 6: Membrane potential as a function of the natural logarithm of the ratio of $\mathrm{NaCl}$ concentrations

Figure 7: Nyquist plot for $\mathrm{NaCl}$ solution $1 \mathrm{~mol} / \mathrm{m} 3$. The best fitting according to Equation (A.1) is shown as a solid line. Some frequencies are superimposed.

Figure 8: a) Permittivity inside membrane pores along with the global one for the wet membrane as a function of concentration. The dependence of permittivity on the membrane thickness is also included by showing the $\pm 30 \mathrm{~nm}$ dashed lines. For the wet membrane, the corresponding lines have not been drawn to avoid unnecessary complications in the figure. The results for $\varepsilon_{\mathrm{p}}$ inside the pores obtained by fitting the SEDE-VCh model are also shown as a dotted line. b) Conductivity inside the pores and for the wet membrane, as function of concentration.

Figure 9: Fitting of Equation (9) showing $\kappa_{\mathrm{p}} / \kappa_{\mathrm{b}}$ as a function of concentration.

Figure 10: Freundlich isotherms for the volume charge density in the membrane as function of concentration. 
Figure 11: Intrinsic retention as a function of permeate flux for different feed concentrations: Experimental data (symbols) and model predicted values (lines).

Figure 12: Left axis: Deviation in percentage between experimental retention values and intrinsic ones predicted by the model versus the concentration. The line is only an eye guide. Right axis: Product of the inverse Debye length and the pore radius versus concentration. 


\section{Tables Caption}

Table 1: Modeling fixed parameters.

Table 2: Capacities and conductances of the active layer of the membrane.

Table 3: Mobilities ratio and Freundlich parameters as a function of active layer thickness.

Table A.1: Relation between phase and dielectic parameters[58].

Table B.1: SEDE-VCh equations for slit shaped pores. 


\section{Tables}

Table 1: Modeling fixed parameters.

\begin{tabular}{cc}
\hline \multicolumn{2}{c}{ MEMBRANE PARAMETERS } \\
\hline$L_{\mathrm{w}}$ & $(2.78 \pm 0.05) \cdot 10^{-11} \mathrm{~m} / \mathrm{s} \cdot \mathrm{Pa}$ \\
$\varepsilon_{\mathrm{d}}$ & 3 \\
$\Delta x_{\mathrm{a}}$ & $90 \pm 30 \mathrm{~nm}$ \\
$t_{1, \mathrm{p}}$ & $0.72 \pm 0.03$ \\
$r_{\mathrm{p}}$ & $0.46 \pm 0.08 \mathrm{~nm}$ \\
$\eta_{\mathrm{p} \mathrm{slit}}$ & $(5.4 \pm 0.6) \cdot 10^{-3} \mathrm{~Pa} \cdot \mathrm{s}$ \\
$\Delta x_{\mathrm{a}} / A_{\mathrm{ka}}$ & $(4.7 \pm 0.8) \cdot 10^{-7} \mathrm{~m}$ \\
$A_{\mathrm{ka}}$ & $0.19 \pm 0.09$ \\
\hline
\end{tabular}

Table 2: Capacities and conductances of the active layer of the membrane.

\begin{tabular}{cccccccc}
\hline & \multicolumn{7}{c}{ Concentration $\left(\mathrm{mol} / \mathrm{m}^{3}\right)$} \\
\cline { 2 - 8 } & 0.01 & 0.02 & 0.1 & 0.2 & 1 & 2 & 10 \\
\hline$C_{\text {memb }}(\mathrm{nF})$ & $990 \pm 30$ & $990 \pm 30$ & $790 \pm 20$ & $700 \pm 20$ & $600 \pm 18$ & $610 \pm 18$ & $610 \pm 18$ \\
$\mathrm{G}_{\text {memb }}(\mu \mathrm{S})$ & $345 \pm 10$ & $374 \pm 11$ & $513 \pm 15$ & $840 \pm 30$ & $4590 \pm 160$ & $9600 \pm 300$ & $49700 \pm 1500$ \\
\hline
\end{tabular}

Table 3: Mobilities ratio and Freundlich isotherm parameters as a function of active layer thickness.

\begin{tabular}{cccc}
\hline$\Delta x_{\mathrm{a}}(\mathrm{nm})$ & $\left(U_{1, \mathrm{p}} / U_{1, \mathrm{~b}}\right) \cdot 10^{2}$ & $r \cdot 10^{3}\left(\mathrm{~mol} / \mathrm{m}^{3}\right)$ & $\Gamma$ \\
\hline 120 & $10.3 \pm 0.2$ & $9.10 \pm 0.05$ & $1.02 \pm 0.06$ \\
\hline 90 & $2.43 \pm 0.08$ & $29.3 \pm 0.1$ & $0.870 \pm 0.009$ \\
\hline 60 & $1.48 \pm 0.06$ & $30.9 \pm 0.1$ & $0.738 \pm 0.008$ \\
\hline
\end{tabular}


Table A.1. Relation between phase and dielectic parameters [58].

The sub-indexes $\mathrm{h}$ and $\mathrm{l}$ refer to the high and low frequencies and memb and $\mathrm{b}$ refer to inside and outside of each membrane system layer.

$\frac{\text { Table B.1: SEDE-VCh equations for slit shaped pores. }}{\text { TRANSPORT EQUATIONS INSIDE OF THE PORE }}$

$$
\begin{aligned}
& \frac{d c_{i}}{d x}=\frac{J_{V}}{K_{i, \mathrm{~d}} D_{i, \infty} A_{\mathrm{ka}}}\left(K_{i, c}^{\prime} c_{i}-c_{i, \mathrm{perm}}\right)-\frac{z_{i} F}{R T} \frac{d \psi}{d x} \\
& \sum_{i} c_{i} z_{i}+Y_{c_{2}}{ }^{\Gamma}=0 \\
& \frac{d \psi}{d x}=\frac{\sum_{i}\left(\frac{z_{i} J_{V}}{K_{i, \mathrm{~d}} D_{i, \infty} A_{\mathrm{ka}}}\right)\left(K_{i, c}^{\prime} c_{i}-c_{i, \mathrm{perm}}\right)+\left(\frac{J_{V} \Gamma Y c_{2}{ }^{\Gamma-1}}{K_{i, \mathrm{~d}} D_{2, \infty} A_{\mathrm{ka}}}\right)\left(K_{2, c}^{\prime} c_{2}-c_{2, \mathrm{perm}}\right)}{\frac{F}{R T}\left(\sum_{i} c_{i} z_{i}^{2}+z_{2} Y_{c_{2}}{ }^{\Gamma}\right)}
\end{aligned}
$$




$$
\begin{array}{ll} 
& c_{i}(x=0)=c_{i, 0} \\
& c_{i}(x=\Delta x)=c_{i, \Delta x_{a}}
\end{array}
$$

$$
K_{i, \mathrm{~d}}=\frac{1+\frac{9}{16} \lambda_{i} \ln \lambda_{i}-1.19358 \lambda_{i}+0.4285 \lambda_{i}^{3}-0.3192 \lambda_{i}^{4}+0.08428 \lambda_{i}^{5}}{1-\lambda_{i}}
$$

$$
K_{i, \mathrm{c}}=\frac{1-3.02 \lambda_{i}^{2}+5.776 \lambda_{i}^{3}-12.3675 \lambda_{i}^{4}+18.9975 \lambda_{i}^{5}-15.2185 \lambda_{i}^{6}+4.8525 \lambda_{i}^{7}}{1-\lambda_{i}}
$$

$$
K_{i, \mathrm{c}}^{\prime}=K_{i, \mathrm{c}}+\left(2-\phi_{i}\right) K_{i, \mathrm{~d}}\left(\frac{2 \lambda_{i}}{3}\right)
$$

\section{PARTITIONING EQUATIONS}

$$
\frac{c_{i, 0}}{c_{i, m}}=\theta_{i}(m \square 0) \text { and } \frac{c_{i, \Delta x_{a}}}{c_{i, p}}=\theta_{i}\left(\operatorname{perm} \square \Delta x_{a}\right)
$$

$(m \square 0)$ and $\left(\right.$ perm $\left.\square \Delta x_{a}\right)$ correspond to the feed side and permeate side, respectively, of the active layer of the membrane.

\section{THE DIELECTRIC BORN ENERGY}

$$
\Delta W_{i, \text { Born }}^{\prime}=\frac{\left(z_{i} e\right)^{2}}{8 \pi k_{B} T \varepsilon_{0} a_{s}}\left(\frac{1}{\varepsilon_{\mathrm{p}}}-\frac{1}{\varepsilon_{\mathrm{b}}}\right)
$$

Where $a_{s}$ is the cavity radius defined by [61] as the distance from the center of the ion to the point where the relative permittivity becomes different than the vacuum one, $\varepsilon_{0}$.

\section{THE DIELECTRIC IMAGE FORCE ENERGY}

$$
\begin{aligned}
& \Delta W_{i, \text { im }}^{\prime}=-\frac{r_{\mathrm{B}}}{r_{\mathrm{p}}} \ln \left[1-\left(\frac{\varepsilon_{\mathrm{p}}-\varepsilon_{\mathrm{d}}}{\varepsilon_{\mathrm{p}}+\varepsilon_{\mathrm{d}}}\right) \exp (-2 \mu)\right] \\
& r_{B}=\frac{\left(z_{i} F\right)^{2}}{8 \pi \varepsilon_{0} \varepsilon_{\mathrm{p}} R T N_{\mathrm{A}}} \\
& \mu=\frac{r_{p}}{\lambda_{0}} \sqrt{\frac{I_{\mathrm{b}}}{I_{\mathrm{p}}}}
\end{aligned}
$$

Where $r_{B}$ is the Bjerrum radius, $\lambda_{0}$ is the Debye length. $I_{\mathrm{b}}$ and $I_{\mathrm{p}}$ are the ionic strength outside and inside the pores respectively. 


$$
R_{i, \mathrm{nt}}=1-\frac{c_{i, \mathrm{perm}}}{c_{i, \mathrm{~m}}}
$$


Electrodes

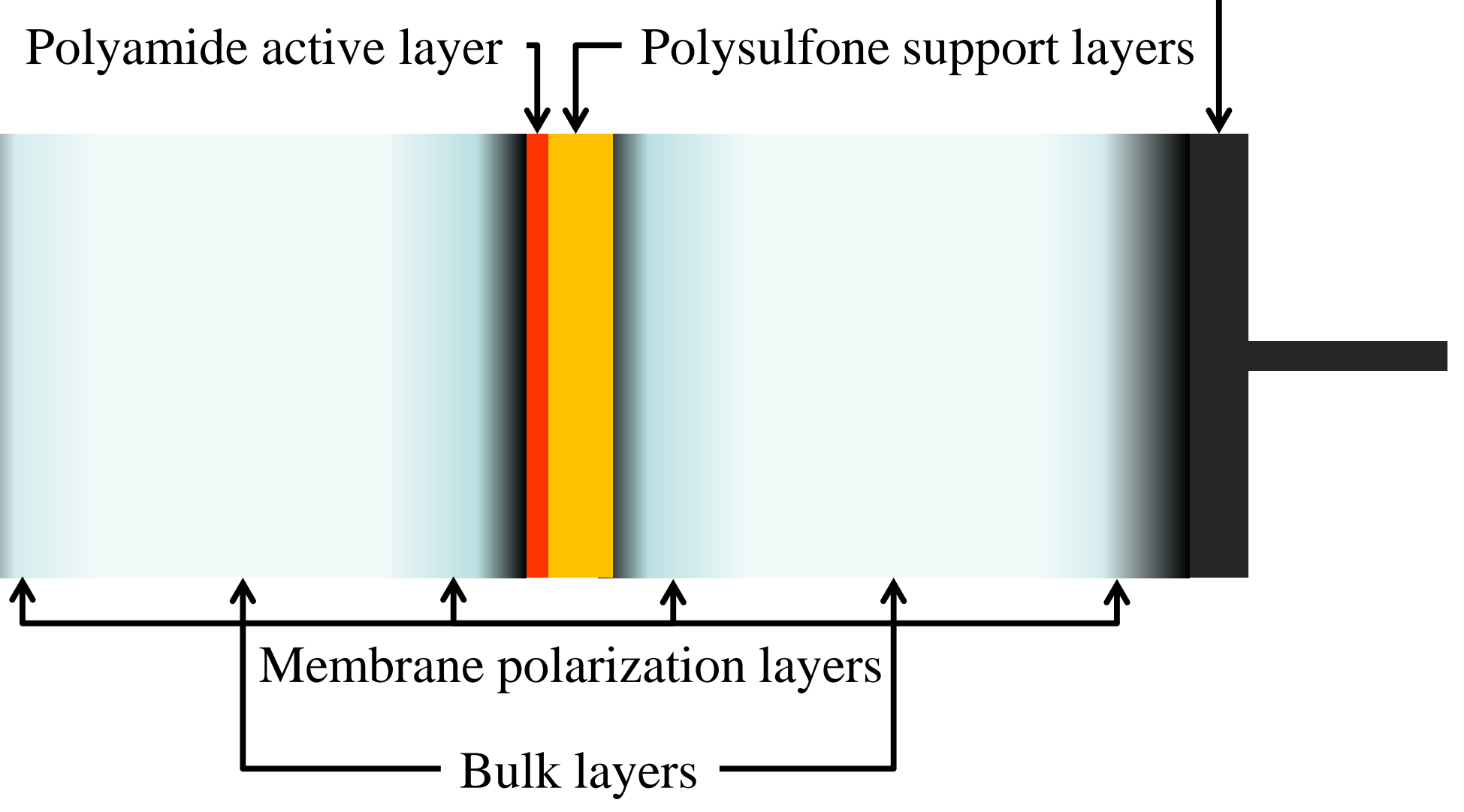

Electrode polarization layers 


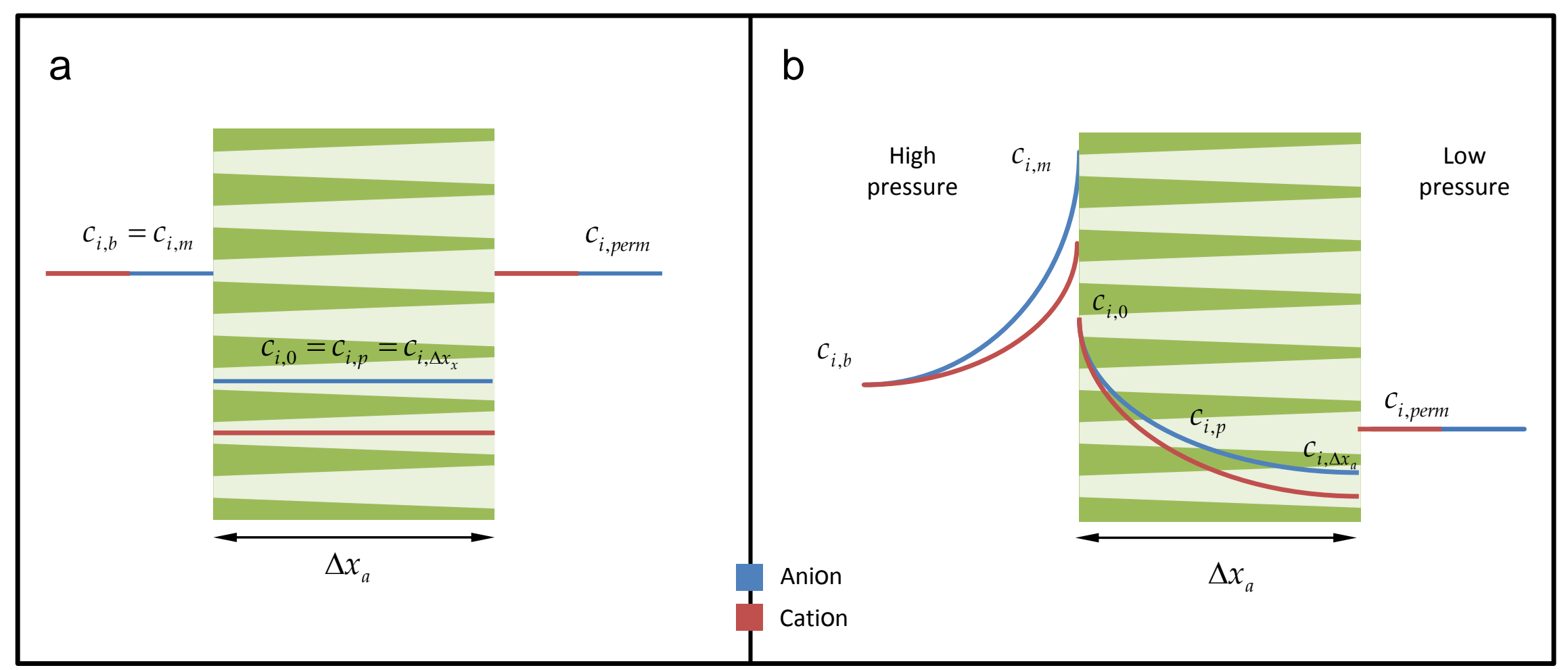

Figure 2 


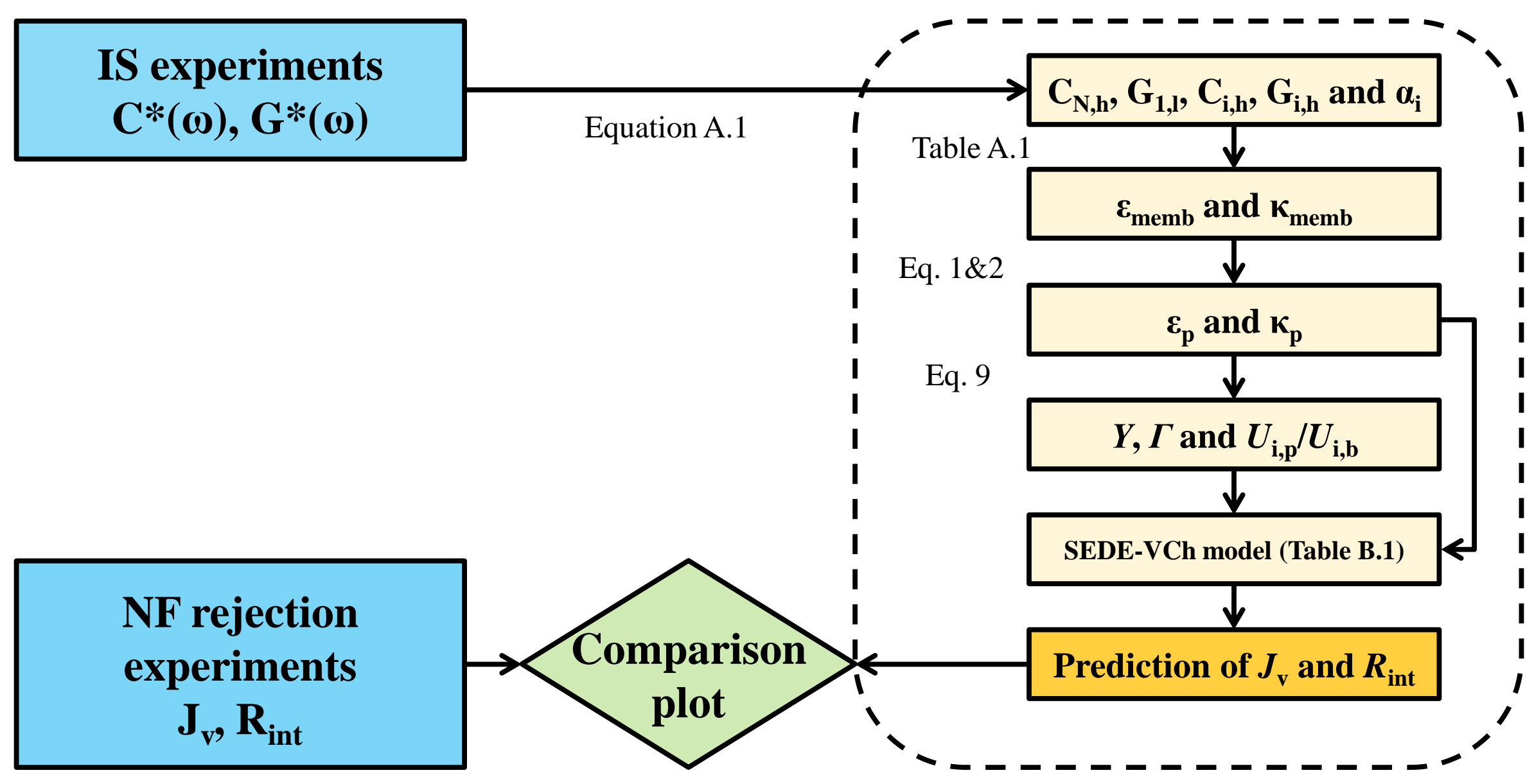

Figure 3 


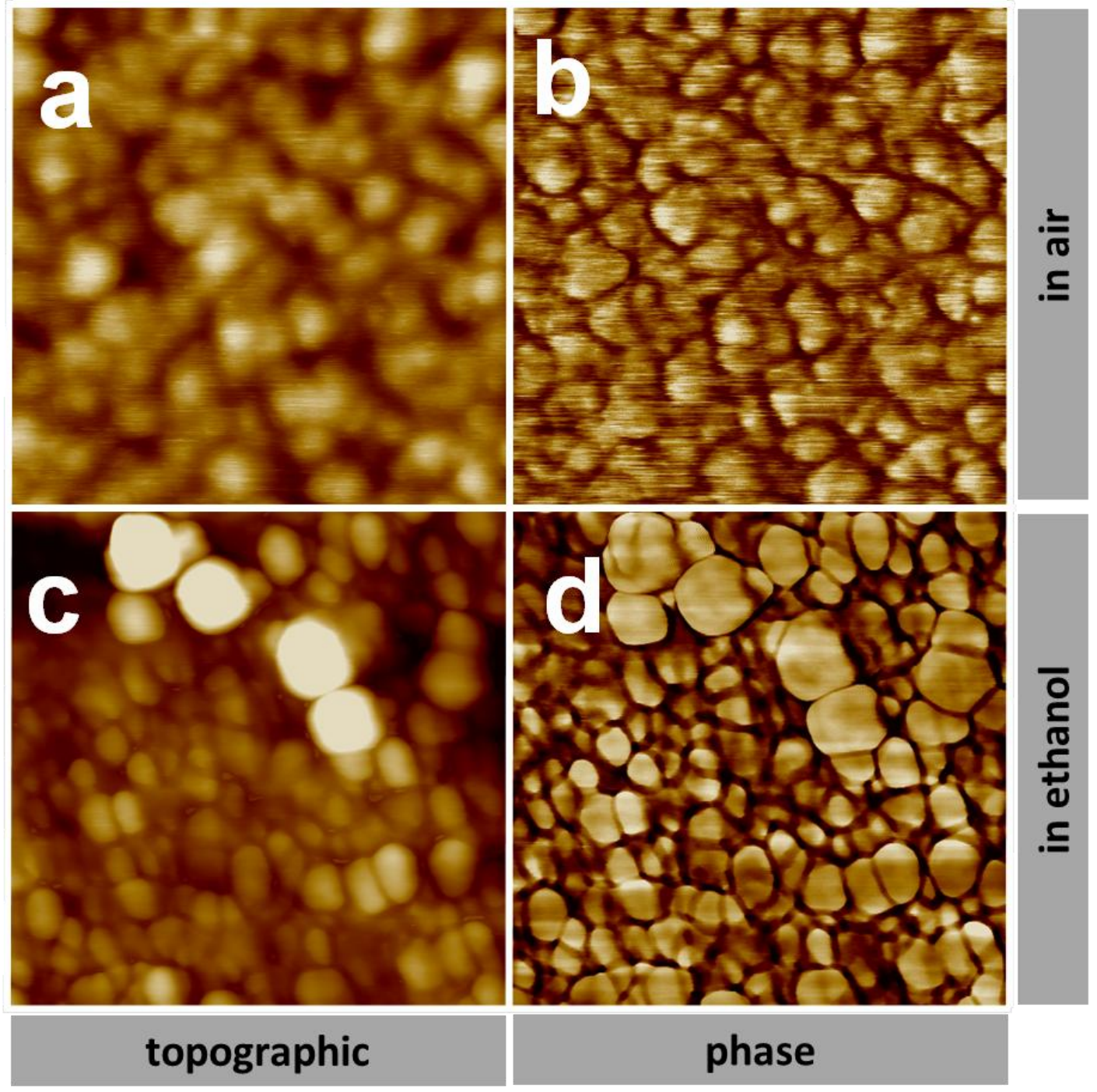

Figure 4 


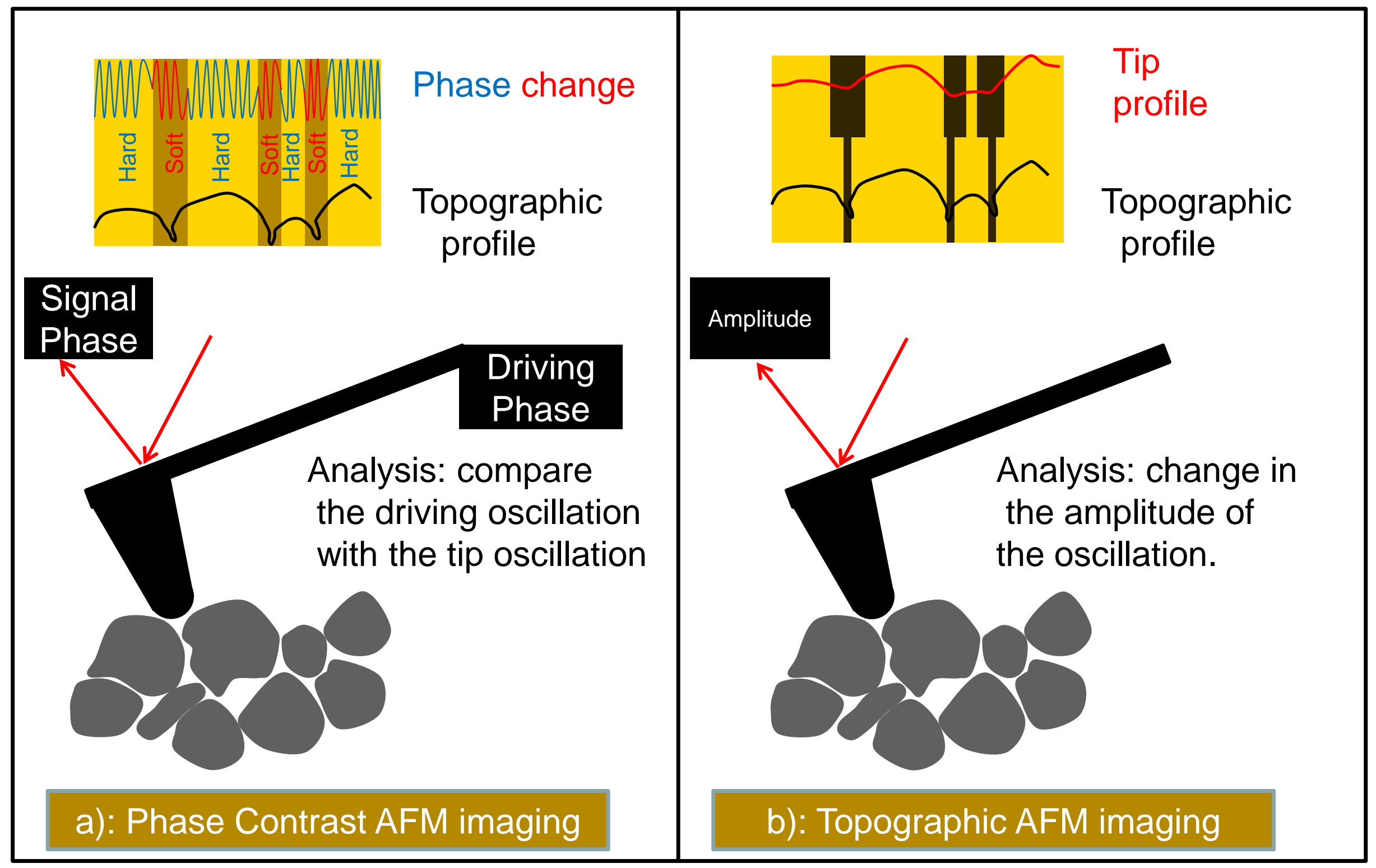

Figure 5 


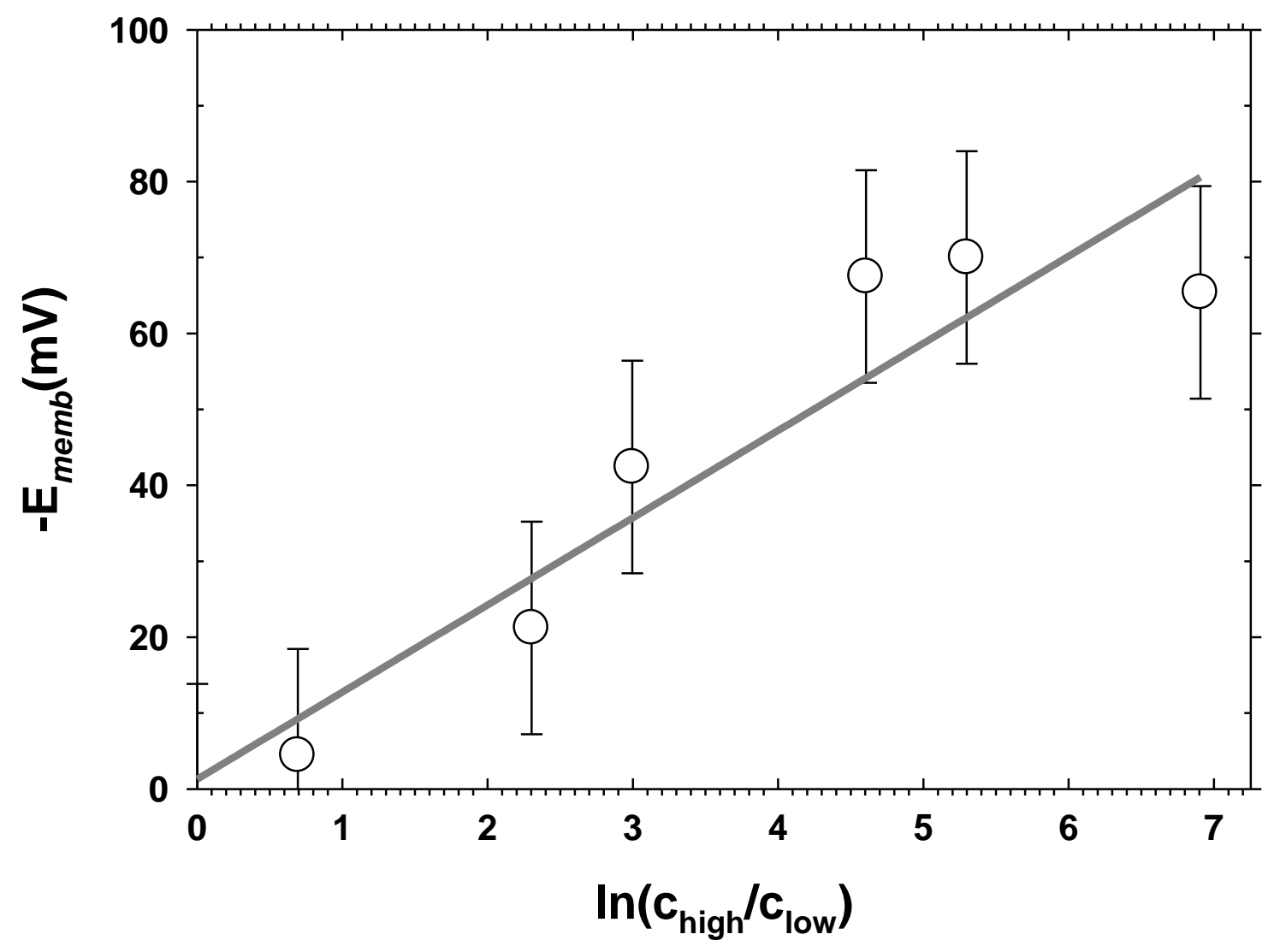

Figure 6 


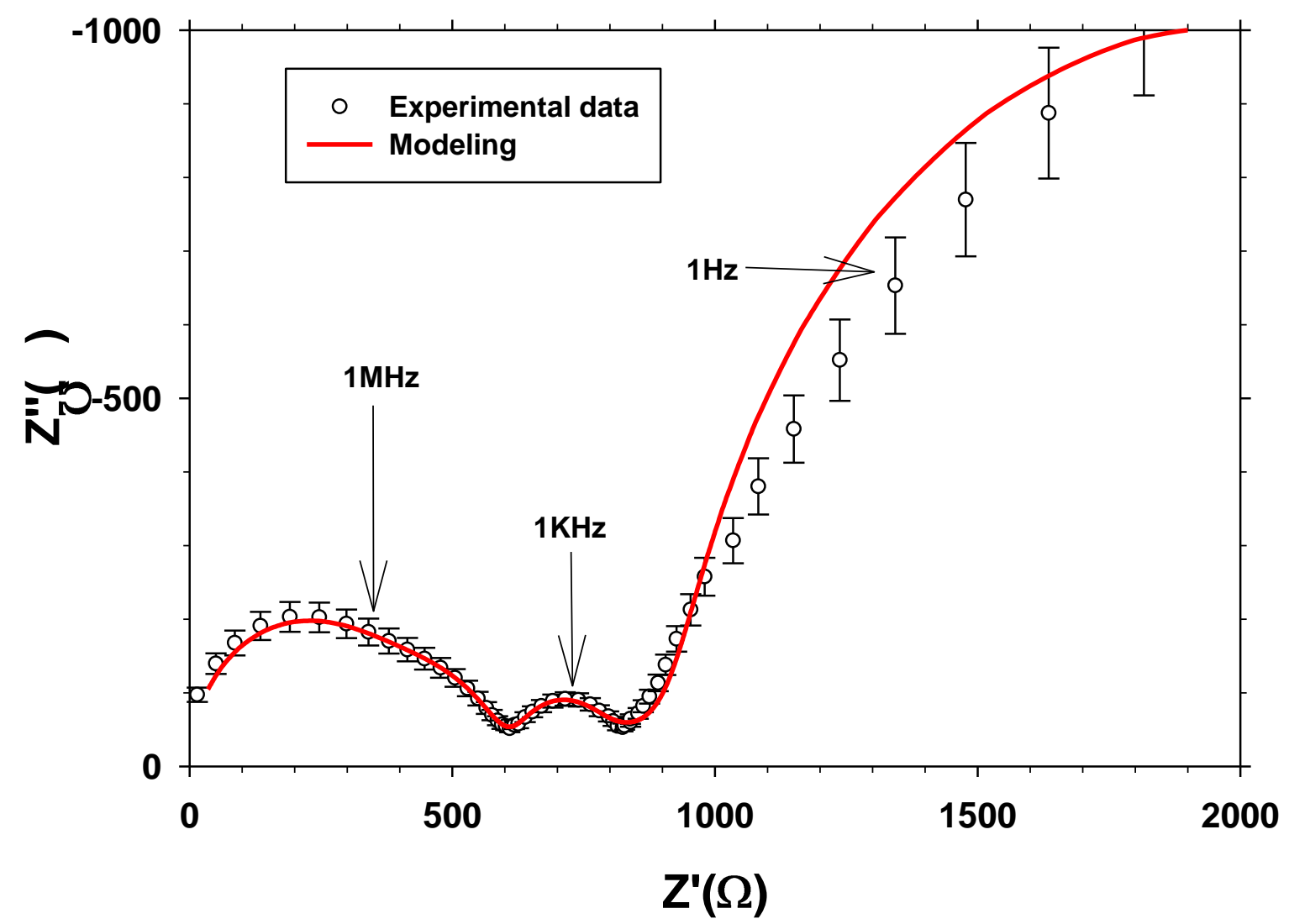

Figure 7 
a

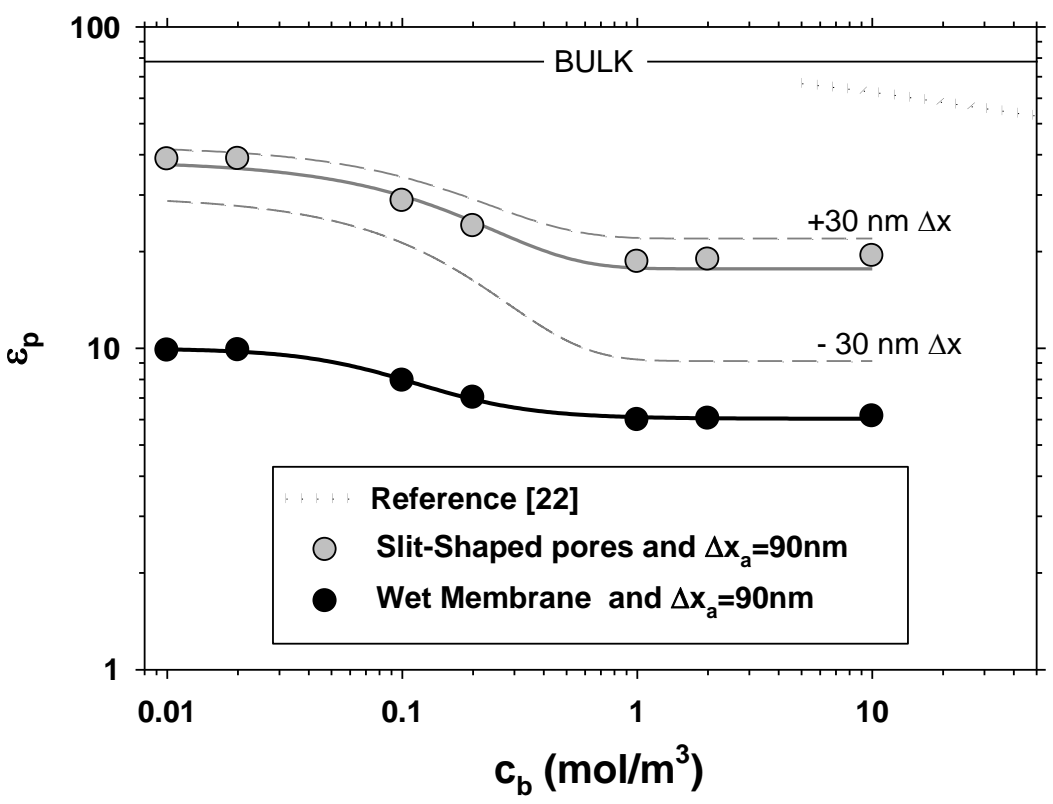

b

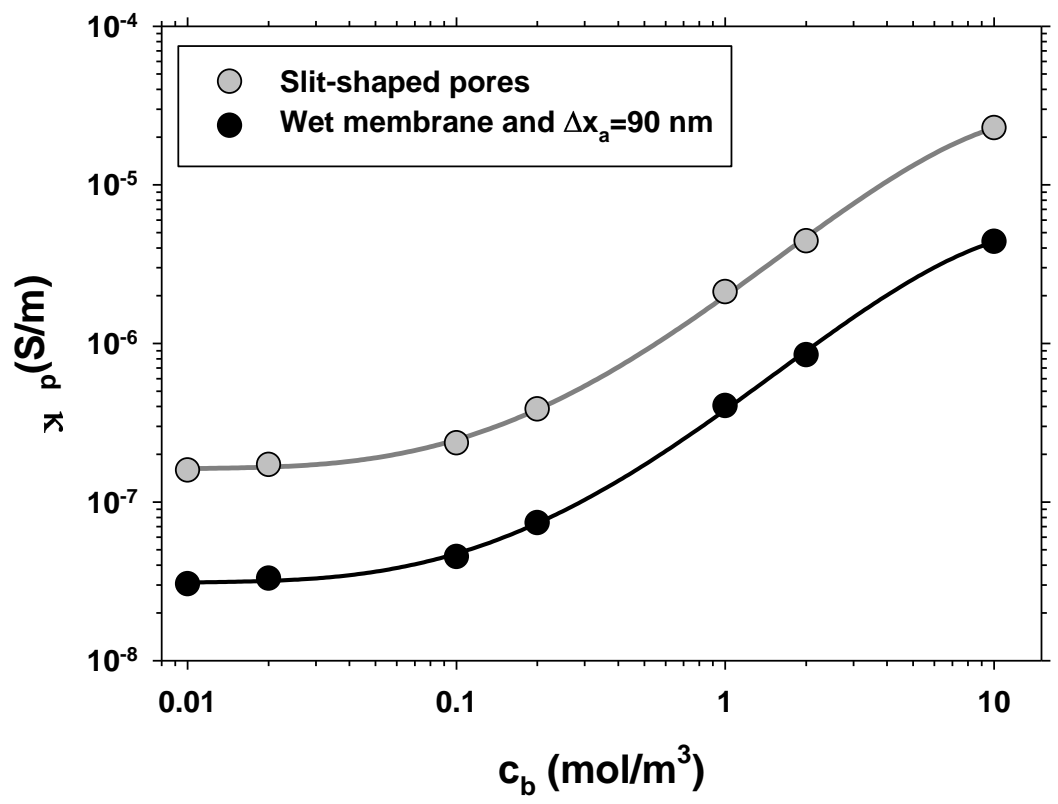

Figure 8 


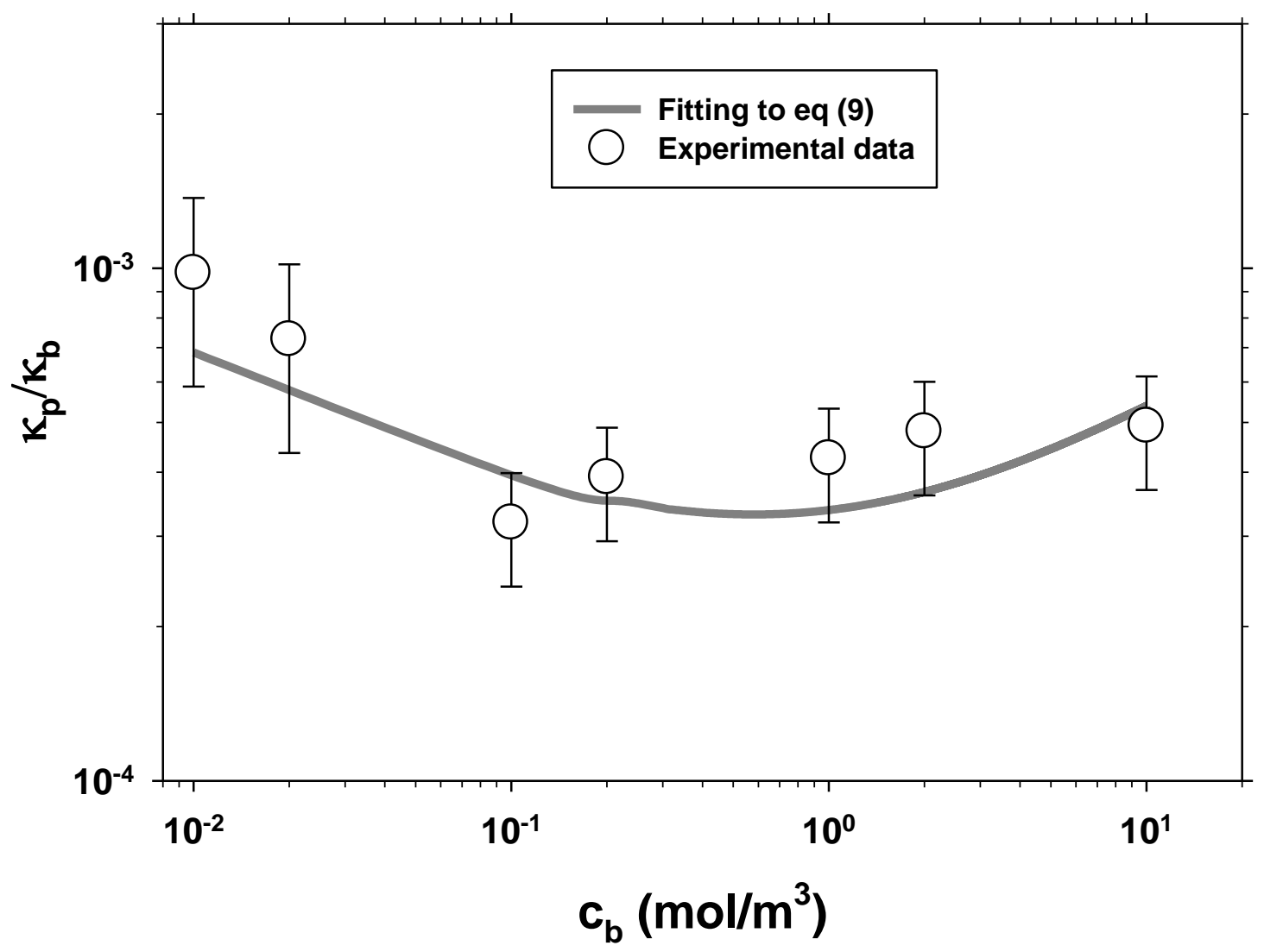

Figure 9 


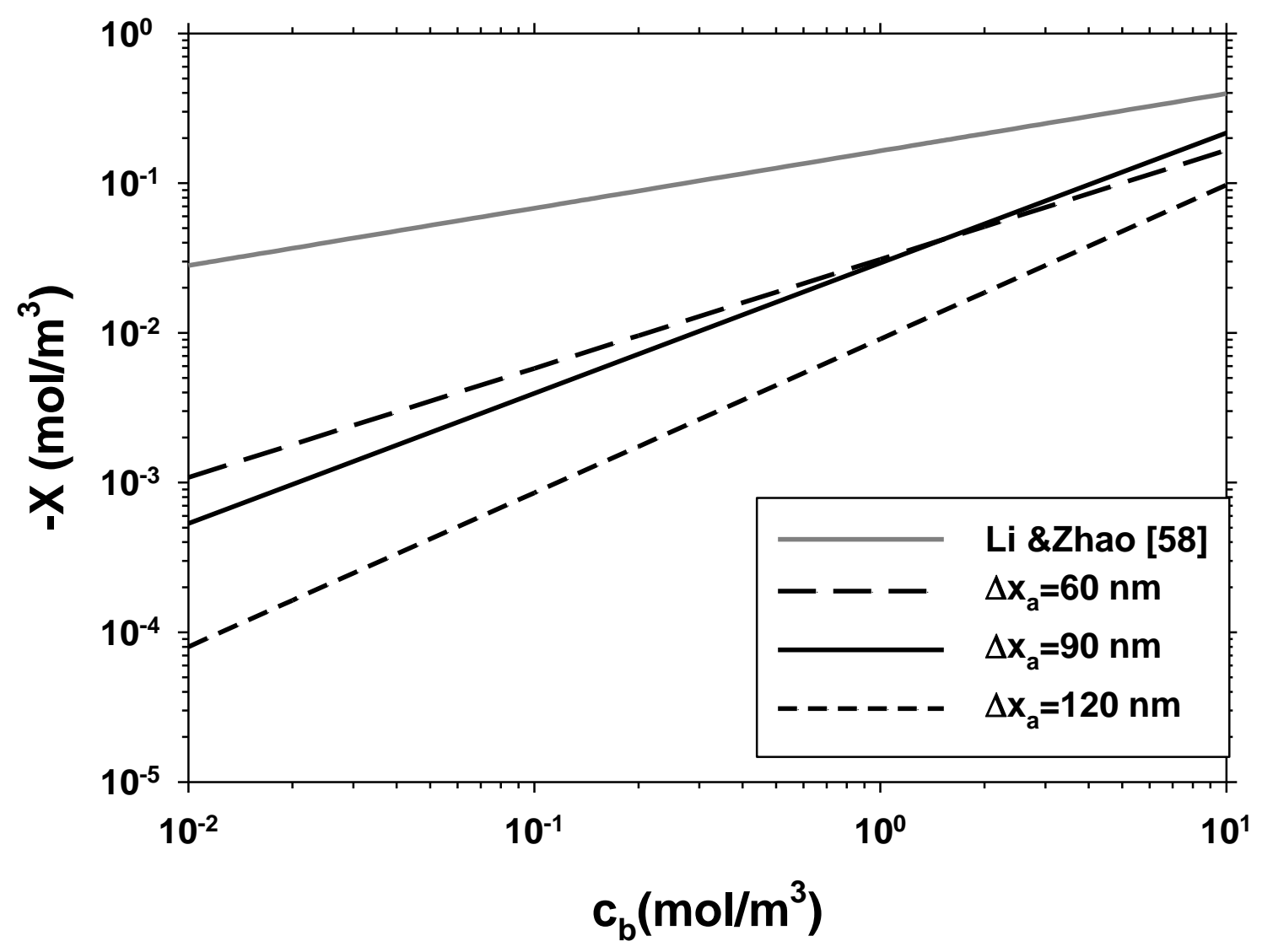

Figure 10 


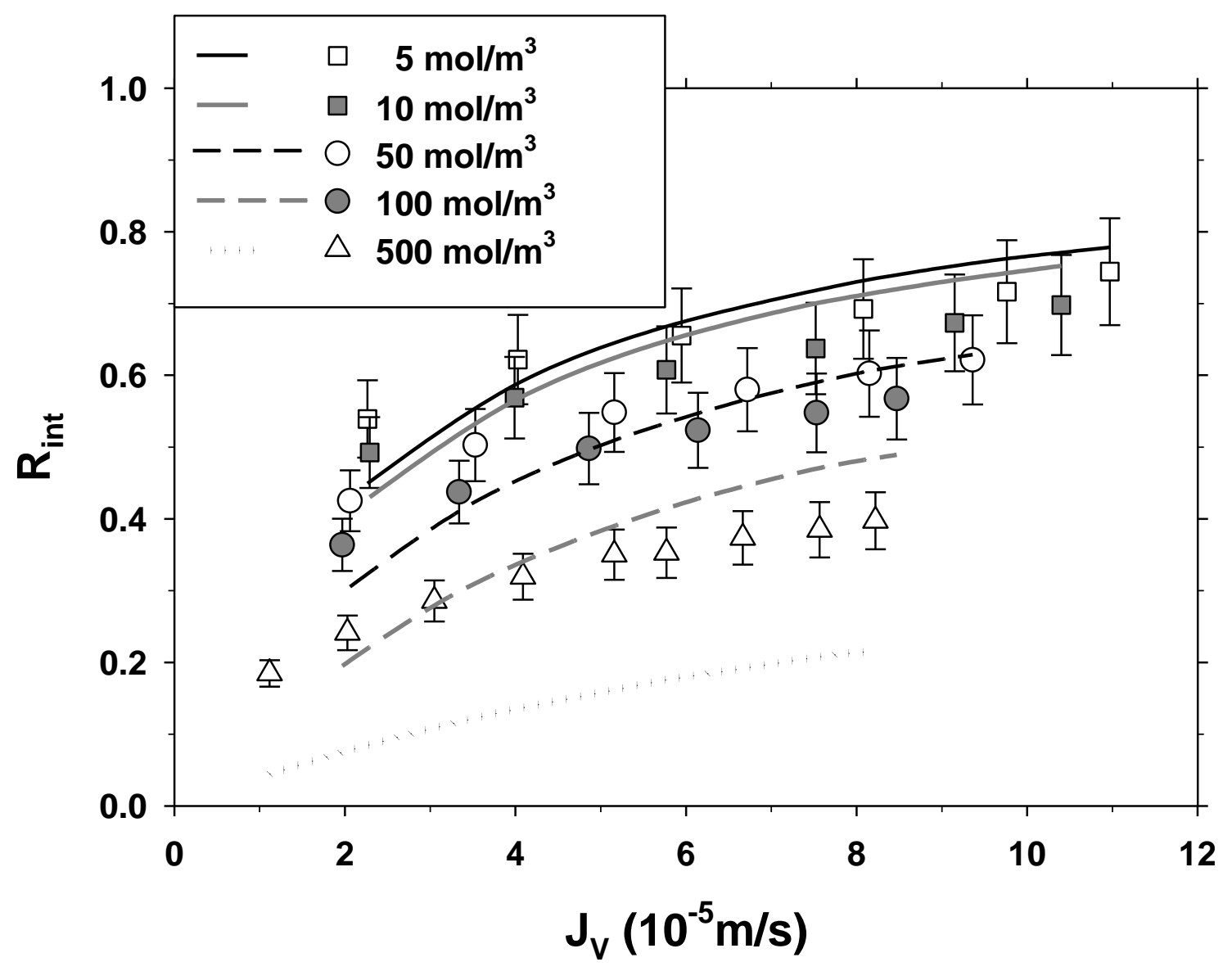

Figure 11 


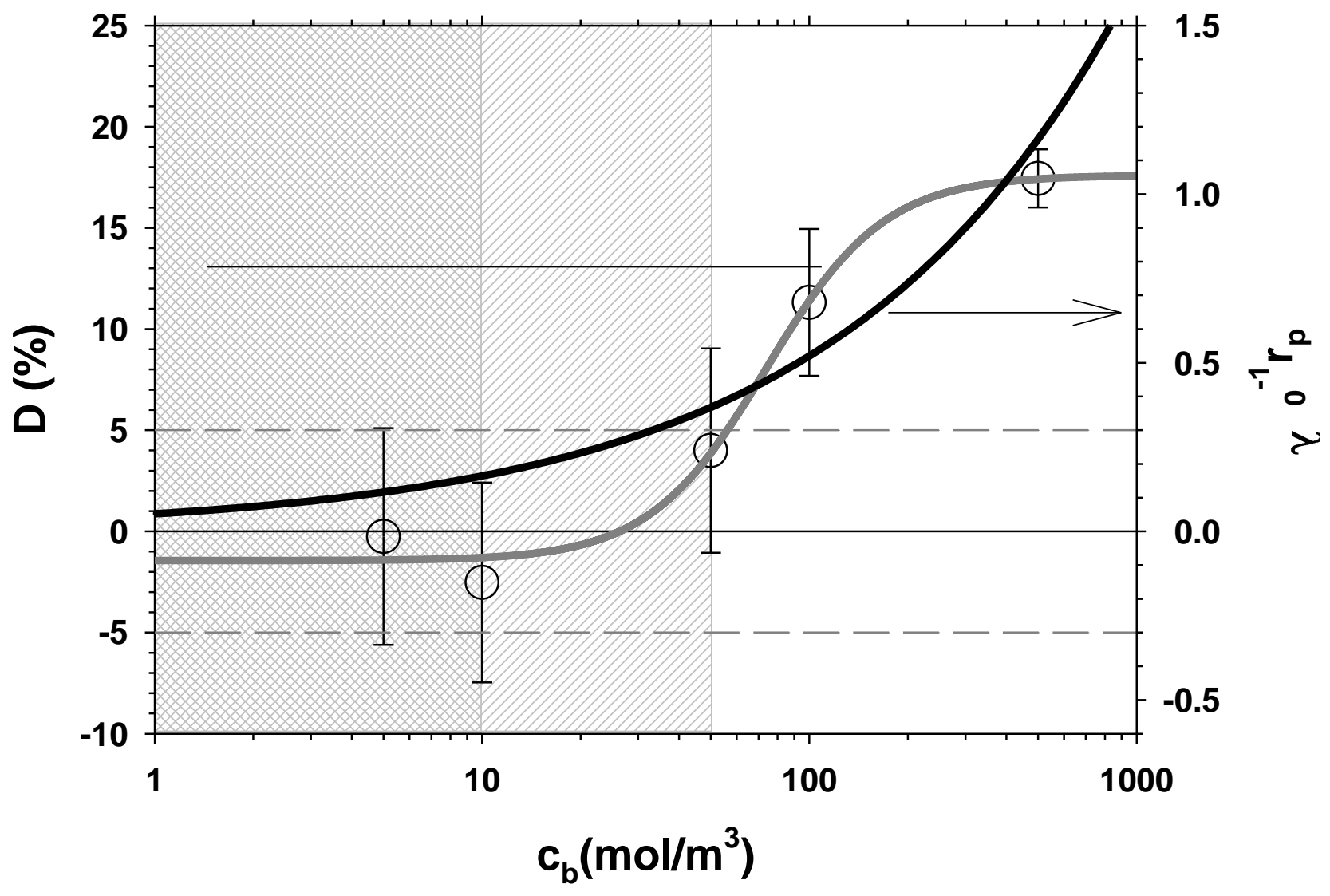

Figure 12 


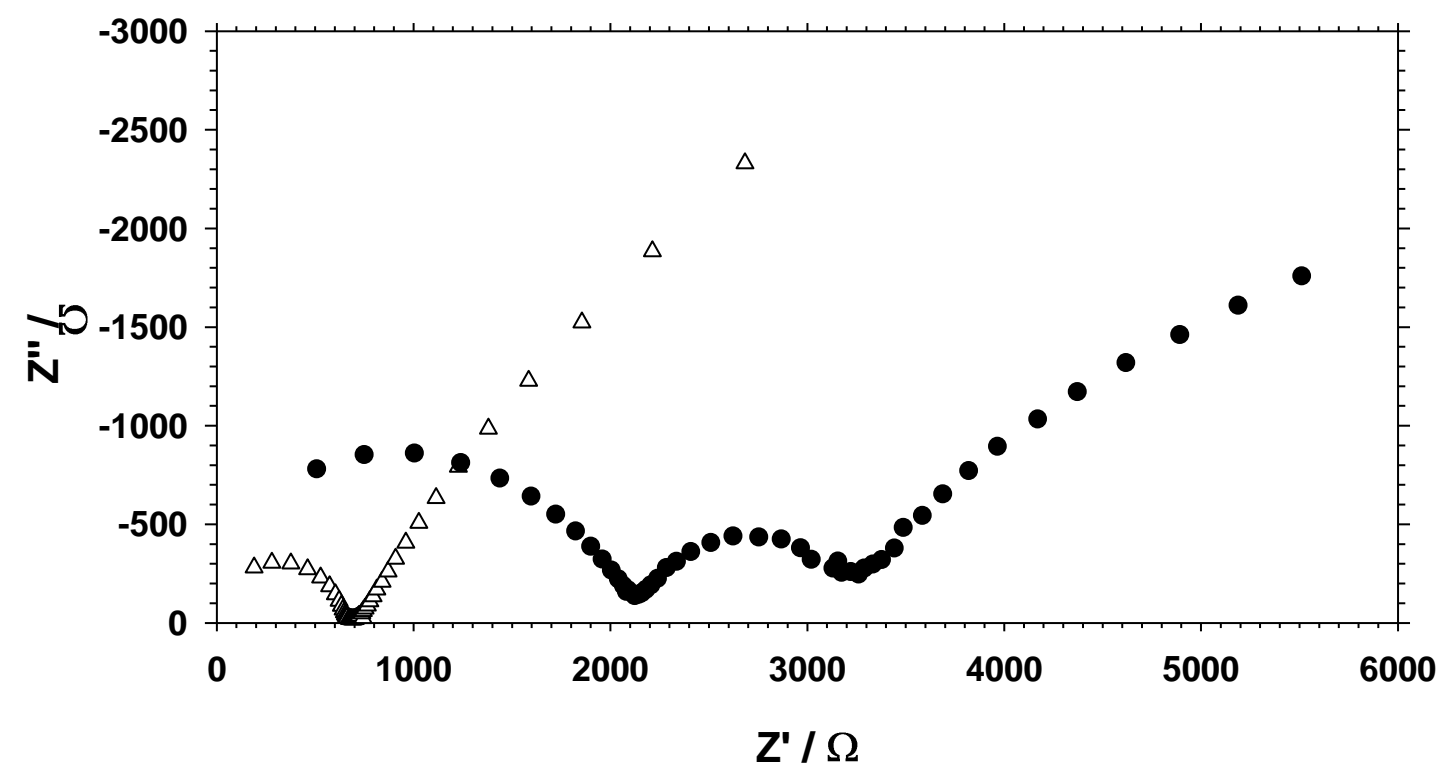

S-1: Nyquist diagram for $0.1 \mathrm{~mol} / \mathrm{m}^{3}$ of $\mathrm{NaCl}$ solution. Black dots represent the measurements with membrane while white dots correspond to the measurements without membrane. 